\title{
A Sound and Complete Abstraction for Reasoning about Parallel Prefix Sums*
}

\author{
Nathan Chong Alastair F. Donaldson Jeroen Ketema \\ Imperial College London \\ $\{$ nyc04,afd,jketema\}@imperial.ac.uk
}

\begin{abstract}
Prefix sums are key building blocks in the implementation of many concurrent software applications, and recently much work has gone into efficiently implementing prefix sums to run on massively parallel graphics processing units (GPUs). Because they lie at the heart of many GPU-accelerated applications, the correctness of prefix sum implementations is of prime importance.

We introduce a novel abstraction, the interval of summations, that allows scalable reasoning about implementations of prefix sums. We present this abstraction as a monoid, and prove a soundness and completeness result showing that a generic sequential prefix sum implementation is correct for an array of length $n$ if and only if it computes the correct result for a specific test case when instantiated with the interval of summations monoid. This allows correctness to be established by running a single test where the input and result require $O(n \lg (n))$ space. This improves upon an existing result by Sheeran where the input requires $O(n \lg (n))$ space and the result $O\left(n^{2} \lg (n)\right)$ space, and is more feasible for large $n$ than a method by Voigtländer that uses $O(n)$ space for the input and result but requires running $O\left(n^{2}\right)$ tests. We then extend our abstraction and results to the context of data-parallel programs, developing an automated verification method for GPU implementations of prefix sums. Our method uses static verification to prove that a generic prefix sum implementation is data race-free, after which functional correctness of the implementation can be determined by running a single test case under the interval of summations abstraction.

We present an experimental evaluation using four different prefix sum algorithms, showing that our method is highly automatic, scales to large thread counts, and significantly outperforms Voigtländer's method when applied to large arrays.
\end{abstract}

Categories and Subject Descriptors F.3.1 [Logics and Meanings of Programs]: Specifying, Verifying \& Reasoning about Programs

Keywords Parallel prefix sum computation; GPUs; abstraction; formal verification.

\footnotetext{
${ }^{*}$ This work was supported by the EU FP7 STREP project CARP (project number 287767), the EPSRC PSL project (EP/I006761/1), and an EPSRC First Grant (EP/K011499/1).
}

Permission to make digital or hard copies of all or part of this work for personal or classroom use is granted without fee provided that copies are not made or distributed for profit or commercial advantage and that copies bear this notice and the full citation on the first page. Copyrights for components of this work owned by others than ACM must be honored. Abstracting with credit is permitted. To copy otherwise, or republish, to post on servers or to redistribute to lists, requires prior specific permission and/or a fee. Request permissions from permissions@acm.org.

POPL '14, January 22-24, 2014, San Diego, CA, USA.

Copyright $\odot 2014$ ACM 978-1-4503-2544-8/14/01... \$15.00.

http://dx.doi.org/10.1145/2535838.2535882

\section{Introduction}

The prefix sum operation, which given an array $A$ computes an array $B$ consisting of all sums of prefixes of $A$, is an important building block in many high performance computing applications. A key example is stream compaction: suppose a group of $n$ threads has calculated a number of data items in parallel, each thread $t$ $(1 \leq t \leq n)$ having computed $d_{t}$ items; now the threads must write the resulting items to a shared array out in a compact manner, i.e. thread $t$ should write its items to a series of $d_{t}$ indices of out starting from position $d_{1}+\cdots+d_{t-1}$. Compacting the data stream by serialising the threads, so that thread 1 writes its results, followed by thread 2, etc., would be slow. Instead, compaction can be performed in parallel via a prefix sum. Each thread $t$ writes its count $d_{t}$ to an array count at position $t$, then the threads perform an exclusive parallel prefix sum (defined formally in Section 2) on count to yield an array index. The prefix sum sets the elements of index to $\left[0, d_{1}, d_{1}+d_{2}, \ldots, d_{1}+\cdots+d_{n-1}\right]$, so that a thread $t$ can write its results to out compactly starting from position index $[t]$.

Stream compaction is just one example; the prefix sum operation is defined for any binary associative operator, and prefix sums using various operators have found wide application in computationally intensive tasks. A selection of examples are summarised in Table 1. For several decades the design of efficient parallel prefix sums has been an active research area. Parallel prefix sums were first implemented in hardware circuits as logic for propagating carry bits in adders [34]. The design space for these circuits has been explored and important circuits known in the literature are due to Kogge and Stone [21], Ladner and Fischer [22], and Brent and Kung [5]. More recent work by Sheeran [33] has further explored the design space. In software, prefix sum algorithms have been used in the context of evaluating polynomials in parallel [36] and Blelloch [3] has proposed the use of prefix sums as a primitive parallel operation, giving numerous examples of their application [4]. Prefix sums are now primitives in parallel programming models such as MPI [29] and OpenMP [35].

Recently there has been a great deal of interest in implementing efficient prefix sums for acceleration on massively parallel graphics processing units (GPUs) [2, 7, 12, 16, 26, 31], implemented using GPU programming models such as OpenCL [19] and CUDA [27]. The major benchmark suites for general-purpose GPU programming, SHOC [12], Rodinia [7] and Parboil [37] all include a variety of prefix sum implementations. The thrust ${ }^{1}$ and CUDA Data Parallel Primitives $(c u d p p)^{2}$ libraries implement efficient prefix sum primitives for NVIDIA GPUs.

We present a method for formally verifying that a generic parallel prefix sum implementation-designed to work for any data

\footnotetext{
${ }_{1}^{1}$ http://code.google.com/p/thrust/

2 http://code.google.com/p/cudpp/
} 


\begin{tabular}{lll} 
Prefix sum application & Data-type & Operator \\
\hline Stream compaction $[2,16]$ & Int & + \\
Sorting algorithms $[16,30]$ & Int & + \\
Polynomial interpolation [13] & Float & $*^{a}$ \\
Line-of-sight calculation [4] & Int & $\max$ \\
Binary addition [22] & $\begin{array}{l}\text { pairs-of-bits } \\
\text { transition }\end{array}$ & $\begin{array}{c}\text { carry operator } \\
\text { function }\end{array}$ \\
Finite state machine simulation [22] & $\begin{array}{l}\text { functions } \\
\text { composition }\end{array}$ & $\begin{array}{c}\text { complion } \\
\end{array}$ \\
\end{tabular}

${ }^{a}$ Floating point multiplication is not actually associative, but is often treated as such in applications where some error can be tolerated.

Table 1. Some applications of parallel prefix sums. Stream compaction requires an exclusive prefix sum; the other applications employ regular prefix sums. The carry operator and functional composition are examples of non-commutative operators.

type with an associative operator-is functionally correct. Because prefix sum implementations are at the heart of many parallel applications, the correctness of these implementations is vital. The challenges of concurrent programming make it especially hard to correctly implement prefix sums for modern architectures such as GPUs. This motivates a dedicated verification method.

By observing that a generic prefix sum algorithm may only exploit the property of associativity, we have devised a novel abstraction which we call the interval of summations abstraction. For $i \leq j$, the interval of summations abstraction represents a contiguous summation interval of input elements in $[i] \oplus \cdots \oplus$ in $[j]$ (with respect to any data type and associative operator $\oplus$ ) abstractly as a pair $(i, j)$. Abstract summation intervals can be added together if they 'kiss': the abstract sum of $(i, j)$ and $(k, l)$ is $(i, l)$ if $j+1=k$; otherwise, the addition results in a special value $\top$ which represents all sums of input elements that are not necessarily contiguous. Summing any monoid element to $\top$ yields $T$, modelling the fact that, using only the property of associativity, a non-contiguous summation of inputs cannot be made contiguous by adding more inputs. We present the interval of summations abstraction as a monoid, with an identity element representing an empty summation. This makes it possible to run a generic prefix sum implementation with respect to the data type and operator defined by the interval of summations monoid.

Our first main contribution is a theorem showing that a sequential generic prefix sum on $n$ elements is correct for all data types and operators if and only if, when instantiated using the interval of summations monoid and applied to the input sequence $[(0,0),(1,1), \ldots,(n-1, n-1)]$, it computes the correct result sequence: $[(0,0),(0,1), \ldots,(0, n-1)]$. This theorem shows that the interval of summations abstraction is sound and complete: by running a single test we can establish either that the generic prefix sum is correct (if the test passes), or that it is incorrect for one specific data-type/operator pair, namely that of the interval of summations monoid itself (if the test fails). Our result provides a highly scalable method for verifying sequential prefix sums: elements of the interval of summations monoid capable of representing intervals of up to length $n$ can be encoded using $O(\lg (n))$ bits, allowing a generic prefix sum to be verified by running a single test case requiring $O(n \lg (n))$ space for both the input and result. This is an improvement on a previous result by Sheeran $[33,38]$ which allows a sequential program implementing a prefix sum to be verified by running one test case requiring $O(n \lg (n))$ space for the input and $O\left(n^{2} \lg (n)\right)$ space for the result. For large values of $n$, this space requirement becomes infeasible. Our method is also more practically feasible than an alternative approach of Voigtländer [38] that uses only $O(n)$ space for the input and result but requires running $O\left(n^{2}\right)$ tests.

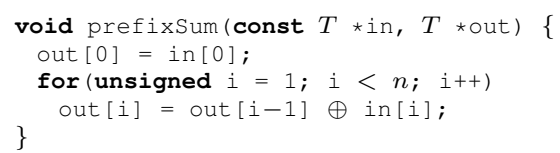

Figure 1. A sequential prefix sum for inputs of length $n$

Our second main contribution is an extension of our method and theoretical results to the case of barrier-synchronising data-parallel programs, the programming model of GPU kernels. This is a contribution over previous work on the correctness of parallel prefix sums $[33,38]$ which applies to synchronous parallel hardware described as sequential HASKELL programs, but not to asynchronous concurrent programs. We show that if a data-parallel program implementing a generic prefix sum can be proved free from data races then correctness of the prefix sum can be established by running a single test case using the interval of summations monoid, as in the sequential case. We use this result to design and implement a highly automatic method for verifying parallel prefix sum implementations at the level of GPU kernel source code, using the GPUVerify tool [1] to prove data race-freedom.

We present a large experimental evaluation using four different prefix sum algorithms implemented as OpenCL kernels. We show that race-freedom of these kernels can be proven efficiently for all power-of-two element sizes up to $2^{31}$, and that verifying the kernels by running a single test is very fast: using two NVIDIA GPUs, two Intel CPUs (for which OpenCL is also supported), and an ARM GPU, prefix sums on vectors of up to length $10^{6}$ can be checked in a matter of seconds. We argue that, once race-freedom has been established, the method of Voigtländer [38] can also be used to prove correctness of prefix sum implementations using testing, but show that our method dramatically outperforms Voigtländer's approach, which requires running $O\left(n^{2}\right)$ tests.

Because we can efficiently prove data race-freedom of GPU kernels implementing prefix sums for all conceivably useful sizes, and because verification using our method involves running a single test case, taking no longer than using the prefix sum in practice, we claim that we have made a major step towards solving the problem of verifying GPU implementations of generic prefix sums.

\section{Background on Prefix Sums}

We briefly review the definitions of a prefix sum and an exclusive prefix sum, and give examples of sequential and parallel prefix sum implementations.

Given a set $\mathbb{S}$ with an associative binary operation $\oplus$ (i.e., a semigroup), the prefix sum of a list $\left[s_{1}, s_{2}, \ldots, s_{n}\right]$ of elements of $\mathbb{S}$ is the list:

$$
\left[s_{1}, s_{1} \oplus s_{2}, \ldots, s_{1} \oplus s_{2} \oplus \cdots \oplus s_{n}\right]
$$

consisting of all sums of prefixes, in increasing order of length. For example, if we consider the set of integers under addition, the prefix sum of $[1,3,5,7]$ is $[1,4,9,16]$.

If $(\mathbb{S}, \oplus)$ has an identity element 1 , so that $(\mathbb{S}, \oplus)$ is a monoid, then the exclusive prefix sum of $\left[s_{1}, s_{2}, \ldots, s_{n}\right]$ is defined as:

$$
\left[\mathbf{1}, s_{1}, s_{1} \oplus s_{2}, \ldots, s_{1} \oplus s_{2} \oplus \cdots \oplus s_{n-1}\right] .
$$

For example, if $\mathbb{S}$ is the set of all four-bit binary numbers and $\oplus$ is the bitwise-or operator with identity 0000 then the exclusive prefix sum of $[0001,0010,0100,1000]$ is $[0000,0001,0011,0111]$.

It is trivial to implement a sequential prefix sum: the C-like program of Figure 1 computes the prefix sum of in into out, 


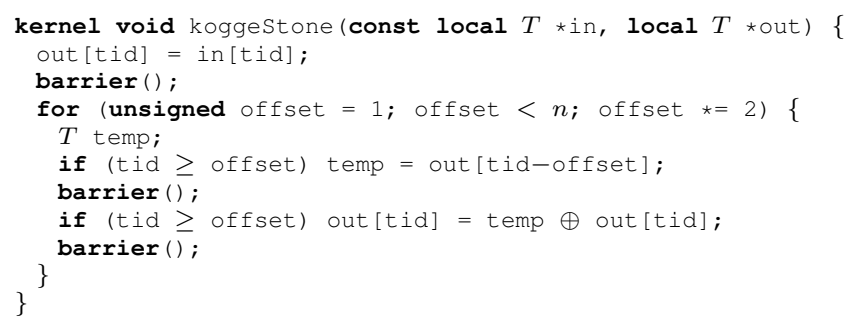

Figure 2. Kogge-Stone prefix sum implemented in OpenCL

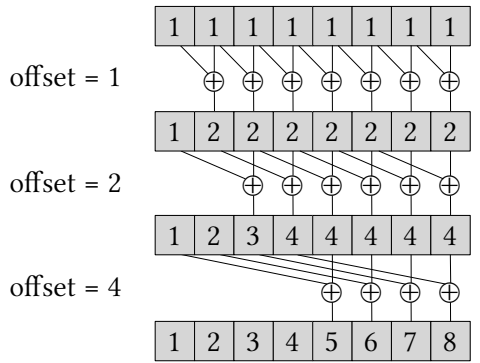

Figure 3. Evolution of the output array for the Kogge-Stone kernel of Figure 2 with $n=8$ for an example input

where $T$ is some data type with associative binary operator $\oplus$. An exclusive prefix sum can be implemented similarly. ${ }^{3}$

Figure 2 shows the Kogge-Stone parallel prefix sum implemented in OpenCL and designed to be executed by a single workgroup of $n$ threads. ${ }^{4}$ The koggeStone function is marked kernel to indicate that it is the entry point for a GPU kernel, and arrays in and out have the local qualifier to indicate that they are allocated in GPU memory local to the work-group. Threads execute asynchronously, and a thread can use tid to access its unique thread identifier, in the range $\{0, \ldots, n-1\}$. Threads synchronise by calling barrier (), which causes all threads to wait until every thread reaches the barrier statement. On each iteration of the loop, every thread whose tid is greater than or equal to of $f$ set sums the value it has already computed with the value previously computed by the thread off set places to the left. Figure 3 illustrates how the output array evolves when the kernel is instantiated using integer addition and applied to the input $[1,1,1,1,1,1,1,1]$, with $n=8$.

\section{Sequential Computational Model}

We present a sequential imperative programming language, and define what it means to implement a correct prefix sum in this language. This provides a simple foundation on which to clearly introduce our interval of summations abstraction, which we do in Section 4. We extend our language, abstraction and theoretical results to apply to data-parallel programs (and in particular GPU kernels) in Section 5.

Syntax and typing The syntax for our language is shown in Figure 4, where $c$ ranges over literal values, $v$ and $A$ over scalar and array variable names, respectively, and $o p$ over an unspecified set of binary operators. Our presentation can be easily extended to cater for operators of other arities.

\footnotetext{
${ }^{3}$ For ease of presentation, we assume throughout that the input length $n$ is hard-coded into the program under consideration. In practice, $n$ would be supplied as a parameter.

${ }^{4}$ For readability, we deviate a little from the precise syntax of OpenCL.
}

\begin{tabular}{|c|c|c|c|}
\hline \multirow{4}{*}{\multicolumn{2}{|c|}{ expr $e$}} & $::=c$ & literal \\
\hline & & $v$ & variable \\
\hline & & $A[e]$ & array element \\
\hline & & $e_{1}$ op $e_{2}$ & operator \\
\hline \multirow[t]{4}{*}{ stmt } & & $::=v:=e$ & variable assignment \\
\hline & & $A\left[e_{1}\right]:=e_{2}$ & array element assignment \\
\hline & & if $(e)\left\{s s_{1}\right\}$ else $\left\{s s_{2}\right\}$ & conditional \\
\hline & & while $(e)\{s s\}$ & loop \\
\hline \multicolumn{3}{|c|}{ stmts $s s::=\varepsilon$} & empty sequence \\
\hline & & $s ; s s$ & sequence \\
\hline
\end{tabular}

Figure 4. Syntax

All variables, arrays and literals are typed (assuming some standard syntax for variable and type declarations, which we omit). Types for scalar variables and literals are drawn from a set of base types $\mathcal{T}$, ranged over by $T$, which includes at least integers and Booleans (denoted Int and Bool respectively)_equipped with the usual literals and operators-and the single-element type Unit. Array variables have type $\operatorname{Array}(T)$ which denotes all maps of type Int $\rightarrow T$. For ease of presentation we assume no out-of-bounds errors occur and we do not allow arrays of arrays. The language also omits features such as pointers, procedures and unstructured control flow. In Section 6 we argue that our technique extends to languages with these features.

The typing rules of the language are straightforward and are depicted in Figure 5. As usual there is a context $\Gamma$ which specifies the types of variables.

Operational semantics Let Var be a set of variables and Arr a set of arrays all of which are assumed to be typed. A variable store $\sigma_{\mathrm{v}}$ is a mapping

$$
\operatorname{Var} \rightarrow \biguplus_{T \in \mathcal{T}} T
$$

such that if $v \in \operatorname{Var}$ is of type $T$, then $\sigma_{\mathrm{v}}(v)$ is of type $T$. An array store $\sigma_{\mathrm{A}}$ is a mapping

$$
\operatorname{Arr} \rightarrow \biguplus_{T \in \mathcal{T}} \operatorname{Array}(T)
$$

such that if $A$ is of type $\operatorname{Array}(T)$, then $\sigma_{\mathrm{A}}(A)$ is of type $\operatorname{Array}(T)$.

Expressions are evaluated under a variable store and an array store. Denoting the evaluation of an expression $e$ by $\llbracket e \rrbracket_{\sigma_{\mathrm{A}}}^{\sigma_{\mathrm{V}}}$, we define:

$$
\begin{aligned}
& \llbracket c \rrbracket_{\sigma_{\mathrm{A}}}^{\sigma_{\mathrm{V}}}=c \\
& \llbracket A[e] \rrbracket_{\sigma_{\mathrm{A}}}^{\sigma_{\mathrm{v}}}=\sigma_{\mathrm{A}}(A)\left(\llbracket e \rrbracket_{\sigma_{\mathrm{A}}}^{\sigma_{\mathrm{v}}}\right) \\
& \llbracket v \rrbracket_{\sigma_{\mathrm{A}}}^{\sigma_{\mathrm{v}}}=\sigma_{\mathrm{v}}(v) \\
& \llbracket e_{1} \text { op } e_{2} \rrbracket_{\sigma_{\mathrm{A}}}^{\sigma_{\mathrm{v}}}=\llbracket e_{1} \rrbracket_{\sigma_{\mathrm{A}}}^{\sigma_{\mathrm{v}}} \text { op } \llbracket e_{2} \rrbracket_{\sigma_{\mathrm{A}}}^{\sigma_{\mathrm{v}}}
\end{aligned}
$$

Figure 6 gives the operational semantics for our language. The semantics is defined over program states $\mathcal{S}=\left(\sigma_{\mathrm{v}}, \sigma_{\mathrm{A}}, s s\right)$ with $\sigma_{\mathrm{v}}$ a variable store, $\sigma_{\mathrm{A}}$ an array store and $s s$ a sequence of statements. In the figure, $s s_{1} \cdot s s_{2}$ denotes the concatenation of sequences of statements $s s_{1}$ and $s s_{2}$. The rules are standard for an imperative language like ours, except that we have both a variable and an array store instead of just a single store. Although this split is not strictly needed here, it eases the extension to data-parallel programs in Section 5 where we shall regard variables as thread-local and arrays as shared among all threads.

Let a program $P$ be a sequence of statements. An initial state of $P$ is any program state with $P$ as the sequence of statements. Given an initial state $\mathcal{S}_{0}$ of $P$, an execution of a program $P$ is a finite or infinite sequence:

$$
\mathcal{S}_{0} \rightarrow_{\mathrm{s}} \mathcal{S}_{1} \cdots \rightarrow_{\mathrm{s}} \mathcal{S}_{i} \rightarrow_{\mathrm{s}} \mathcal{S}_{i+1} \rightarrow_{\mathrm{s}} \cdots
$$

with each $\mathcal{S}_{i}(i \geq 0)$ a program state. An execution is maximal if it (a) cannot be extended by applying one of the rules from the 


$$
\begin{aligned}
& \frac{c \text { of type } T}{\Gamma \vdash c: T} \text { (T-Literal) } \quad \frac{v: T \in \Gamma}{\Gamma \vdash v: T}\left(\mathrm{~T} \text {-VARIAble) } \quad \frac{A: \operatorname{Array}(T) \in \Gamma \quad \Gamma \vdash e: \text { Int }}{\Gamma \vdash A[e]: T}\right. \text { (T-ARRAY) } \\
& \frac{o p \text { of type } T_{1} \times T_{2} \rightarrow T_{3} \quad \Gamma \vdash e_{1}: T_{1} \quad \Gamma \vdash e_{2}: T_{2}}{\Gamma \vdash e_{1} \text { op } e_{2}: T_{3}} \text { (T-OP) }
\end{aligned}
$$

(a) Typing rules for expressions

$$
\begin{aligned}
& \frac{v: T \in \Gamma \quad \Gamma \vdash e: T}{\Gamma \vdash v:=e: \text { Unit }} \text { (T-Assign) } \quad \frac{A: \operatorname{Array}(T) \in \Gamma \quad \Gamma \vdash e_{1}: \operatorname{Int} \quad \Gamma \vdash e_{2}: T}{A\left[e_{1}\right]:=e_{2}: \text { Unit }} \text { (T-ArraY-Assign) } \\
& \frac{\Gamma \vdash e: \text { Bool } \Gamma \vdash s s_{1}: \text { Unit } \quad \Gamma \vdash s s_{2}: \text { Unit }}{\Gamma \vdash \text { if }(e)\left\{s s_{1}\right\} \text { else }\left\{s s_{2}\right\}: \text { Unit }} \text { (T-ITE) } \quad \frac{\Gamma \vdash e: \text { Bool } \quad \Gamma \vdash s s: \text { Unit }}{\Gamma \vdash \text { while }(e)\{s s\}: \text { Unit }} \text { (T-Loop) } \\
& \overline{\Gamma \vdash \varepsilon: \text { Unit }}\left(\text { T-EMPTY) } \quad \frac{\Gamma \vdash s: \text { Unit } \quad \Gamma \vdash s s: \text { Unit }}{\Gamma \vdash s ; s s: \text { Unit }}\right. \text { (T-SEQ) }
\end{aligned}
$$

(b) Typing rules for statements

Figure 5. Typing rules of our sequential programming language

$$
\begin{aligned}
& \frac{\sigma_{\mathrm{v}}^{\prime}=\sigma_{\mathrm{v}}\left[v \mapsto \llbracket e \rrbracket_{\sigma_{\mathrm{A}}}^{\sigma_{\mathrm{v}}}\right]}{\left(\sigma_{\mathrm{v}}, \sigma_{\mathrm{A}}, v:=e ; s s^{\prime}\right) \rightarrow_{\mathrm{s}}\left(\sigma_{\mathrm{v}}^{\prime}, \sigma_{\mathrm{A}}, s s^{\prime}\right)}(\mathrm{S} \text {-AsSiGN }) \\
& \frac{\llbracket e_{1} \rrbracket_{\sigma_{\mathrm{A}}}^{\sigma_{\mathrm{v}}}=n \quad A^{\prime}=\sigma_{\mathrm{A}}(A)\left[n \mapsto \llbracket e_{2} \rrbracket_{\sigma_{\mathrm{A}}}^{\sigma_{\mathrm{A}}}\right] \quad \sigma_{\mathrm{A}}^{\prime}=\sigma_{\mathrm{A}}\left[A \mapsto A^{\prime}\right]}{\left(\sigma_{\mathrm{v}}, \sigma_{\mathrm{A}}, A\left[e_{1}\right]:=e_{2} ; s s^{\prime}\right) \rightarrow_{\mathrm{s}}\left(\sigma_{\mathrm{v}}, \sigma_{\mathrm{A}}^{\prime}, s s^{\prime}\right)} \\
& \frac{\llbracket e \rrbracket_{\sigma_{\mathrm{A}}}^{\sigma_{\mathrm{V}}}}{\left(\sigma_{\mathrm{v}}, \sigma_{\mathrm{A}}, \text { if }(e)\left\{s s_{1}\right\} \text { else }\left\{s s_{2}\right\} ; s s^{\prime}\right) \rightarrow_{\mathrm{s}}\left(\sigma_{\mathrm{v}}, \sigma_{\mathrm{A}}, s s_{1} \cdot s s^{\prime}\right)}(\text { S-ITE-T) } \\
& \frac{\neg \llbracket e \rrbracket_{\sigma_{\mathrm{A}}}^{\sigma_{\mathrm{V}}}}{\left(\sigma_{\mathrm{v}}, \sigma_{\mathrm{A}}, \text { if }(e)\left\{s s_{1}\right\} \text { else }\left\{s s_{2}\right\} ; s s^{\prime}\right) \rightarrow_{\mathrm{s}}\left(\sigma_{\mathrm{v}}, \sigma_{\mathrm{A}}, s s_{2} \cdot s s^{\prime}\right)}(\mathrm{S}-\mathrm{ITE}-\mathrm{F}) \\
& \frac{\llbracket e \rrbracket_{\sigma_{\mathrm{A}}}^{\sigma_{\mathrm{V}}}}{\left(\sigma_{\mathrm{v}}, \sigma_{\mathrm{A}}, \text { while }(e)\{s s\} ; s s^{\prime}\right) \rightarrow_{\mathrm{s}}\left(\sigma_{\mathrm{v}}, \sigma_{\mathrm{A}}, s s \cdot \text { while }(e)\{s s\} ; s s^{\prime}\right)}(\text { S-Loop-T) } \\
& \frac{\neg \llbracket e \rrbracket_{\sigma_{\mathrm{A}}}^{\sigma_{\mathrm{V}}}}{\left(\sigma_{\mathrm{v}}, \sigma_{\mathrm{A}}, \text { while }(e)\{s s\} ; s s^{\prime}\right) \rightarrow_{\mathrm{s}}\left(\sigma_{\mathrm{v}}, \sigma_{\mathrm{A}}, s s^{\prime}\right)}
\end{aligned}
$$

Figure 6. Operational semantics of our sequential programming language

operational semantics or (b) is infinite. We say $P$ terminates for an initial state $\mathcal{S}$, if all maximal executions starting from $\mathcal{S}$ are finite. Note that the maximal execution is unique in the current case as execution is deterministic; this will no longer be so in the data-parallel case of Section 5. The proof that our type system and operational semantics satisfy the usual safety property-that types are preserved under execution and progress can always be made unless termination has occurred [28, Section 8.3]—is standard.

Prefix sum algorithms We now define what it means for a program in our language to implement a prefix sum algorithm.

Recall from Section 2 that a prefix sum can be defined with respect to a semigroup, and an exclusive prefix sum with respect to a monoid. Because most prefix sums of interest in practice are over monoids we shall henceforth consider prefix sums over monoids. All the results we present restrict easily to the case of semigroups.

We shall write $M$ to refer to a monoid with elements $\mathbb{S}_{M} \in \mathcal{T}$, binary operator $\oplus_{M}$ (which we assume is a programming language operator) and identity $\mathbf{1}_{M} \in \mathbb{S}_{M}$. Moreover, we say that a variable $v$, respectively an array $A$, is read-only in $P$ if no assignment of the form $v:=\ldots$, respectively $A[e]:=\ldots$, occurs in $P$.

Definition 3.1. Let $M$ be a monoid, $P$ be a program, $n$ a natural number, and in, out arrays of type $\operatorname{Array}\left(\mathbb{S}_{M}\right)$ such that in is read-only in $P$. The program $P$ computes an $M$-prefix sum of length $n$ from in to out for an initial state $\mathcal{S}$ if $P$ terminates for $\mathcal{S}$ and for each final array store $\sigma_{\mathrm{A}}$ it holds that $\sigma_{\mathrm{A}}($ out $)(k)=$ $\bigoplus_{M, 0 \leq i \leq k} \sigma_{\mathrm{A}}($ in $)(i)(0 \leq k<n)$.

The program $P$ implements an $M$-prefix sum of length $n$ from in to out if $P$ computes an $M$-prefix sum of length $n$ from in to out for every initial state.
Whether a program computes a prefix sum for a given input can be established by running the program; determining whether a program implements a prefix sum amounts to functional verification. Implementation of an exclusive prefix sum is defined analogously.

Designating the array in as read-only is for ease of presentation, making it sufficient to consider only the final array store in the above definition and avoiding the need to relate final and initial array stores.

We observe that Definition 3.1 quantifies over all possible final array stores to be able to cover the data-parallel case of Section 5. In the current sequential case the final array store is unique as execution is deterministic.

Henceforth we shall assume that a prefix sum is always from in to out, and shall simply talk about a program implementing an $M$ prefix sum of length $n$, or computing an $M$-prefix sum of length $n$ from an initial state.

\section{The Interval of Summations Abstraction}

We now turn our attention to proving the correctness of generic prefix sums: prefix sums that are designed to be polymorphic, so that they work for any type and operator that together have the properties of a monoid. We present our main theoretical result, that correctness of a generic prefix sum of length $n$ can be established by showing that the prefix sum is correct for one particular monoid, the interval of summations monoid, for one particular input.

Generic prefix sums Let us extend our programming language with a fresh generic type $\mathbb{S}_{X}$, a new operator $\oplus_{X}: \mathbb{S}_{X} \times \mathbb{S}_{X} \rightarrow \mathbb{S}_{X}$, and a distinguished literal value $\mathbf{1}_{X}$ of type $\mathbb{S}_{X}$. We intend this generic type to represent an arbitrary monoid $X=\left(\mathbb{S}_{X}, \oplus_{X}\right)$ with identity $\mathbf{1}_{X}$. 
We call a program that makes use of $\mathbb{S}_{X}$ a generic program. Akin to a generic method in JAVA, a template method in $\mathrm{C}++$, or a function with a type class context in HASKELL, a generic program cannot be directly executed: it must first be instantiated with respect to a specific type.

Definition 4.1. Let $P$ be a generic program and $M$ a monoid. We write $P[M]$ to denote the program that is identical to $P$ except that every occurrence of $\mathbb{S}_{X}, \oplus_{X}, \mathbf{1}_{X}$ is replaced by $\mathbb{S}_{M}, \oplus_{M}, \mathbf{1}_{M}$, respectively. We refer to the process of obtaining $P[M]$ from $P$ as a monoid substitution.

If $M$ already occurs in $P$ then we cannot tell, from $P[M]$ alone, which uses of $M$ were already present in $P$ or originated from uses of $X$. We handle this issue as follows, to simplify our formal treatment: if $M$ occurs in $P$ then we choose a monoid $M^{\prime}$ isomorphic to $M$ such that $M^{\prime}$ does not occur in $P$, and we define $P[M]$ to be $P\left[M^{\prime}\right]$. For ease of presentation we still refer to the monoid $M^{\prime}$ as $M$.

It is easy to see that monoid substitution is well-defined, and that if the abstract program $P$ is well-typed according to the rules of Figure 5 then applying a monoid substitution to $P$ leads to a well-typed program.

Definition 4.2 (Generic prefix sums). Let $P$ be a generic program. Then $P$ implements a generic prefix sum of length $n$ if in and out are of type $\operatorname{Array}\left(\mathbb{S}_{X}\right)$ and, for every monoid $M, P[M]$ implements an $M$-prefix sum of length $n$.

The interval of summations monoid The key insight behind our result is the observation that a generic prefix sum can only rely on the properties of a monoid: associativity and the existence of an identity element. Relying on any additional properties specific to a particular operator, such as commutativity, idempotence or distributivity with respect to some other operator, would render the prefix sum inapplicable in general.

Suppose we wish to compute a prefix sum of length $n$ from array in into array out, with respect to an arbitrary monoid $M$. Thus in and out have type $\operatorname{Array}\left(\mathbb{S}_{M}\right)$, and the prefix sum operator is $\oplus_{M}$. If the prefix sum is correctly implemented then at the end of the computation each element of out must be the sum of a contiguous sequence of elements of in. For example, out [0] should be equal to in[0] and out [3] to in[0] $\oplus_{M}$ in[1] $\oplus_{M}$ in[2] $\oplus_{M}$ in [3]. As computation progresses, these contiguous summations are built up using the $\oplus_{M}$ operator, initially starting with individual elements of in.

Consider, for any $0 \leq k<n$, how out $[k]$ could be computed (to give the main intuition in a simple manner we ignore possible uses of the identity element): either (a) the existing value of a variable or array element could be copied into out $[k]$ or (b) out $[k]$ could be derived by summing two values of $\mathbb{S}_{M}, c$ and $d$, say, using $\oplus_{M}$. In the latter case

- $c$ and $d$ are summations of contiguous elements of in: $c=$ $\bigoplus_{M, 0 \leq j \leq i_{1}}$ in $[j]$ and $d=\bigoplus_{M, i_{2} \leq j \leq k}$ in $[j]$, for some $i_{1}$ and $i_{2}$ (where an empty summation is defined to be $\mathbf{1}_{M}$ ), and

- the summations $c$ and $d$ must 'kiss': we must have $i_{1}+1=i_{2}$.

If $c$ and $d$ did not have these forms then, using only the laws of associativity and identity, it would not be possible to rewrite $c \oplus_{M} d$ into the form $\bigoplus_{M, 0<j<k}$ in $[j]$, the required value for out $[k]$. By a similar argument, if $c$ (similarly $d$ ) is in turn constructed by summing two values $e$ and $f$ then $e$ and $f$ must both be contiguous summations of elements of in that kiss, otherwise it would not be possible, using just the monoid laws, to rearrange $e \oplus_{M} f$ into the form of $c$. Continuing this argument we can see that a correct generic prefix sum must compute its result by successively summing partial sums of contiguous input elements that kiss; if a non-contiguous sum is ever constructed then this sum cannot contribute to the final prefix sum result.

We introduce a monoid, the interval of summations monoid, which captures abstractly the notion of contiguous summation intervals, and the operation of summing pairs of kissing intervals.

Definition 4.3. The interval of summations monoid I (henceforth referred to as the interval monoid) has the elements

$$
\mathbb{S}_{I}=\left\{\left(i_{1}, i_{2}\right) \in \operatorname{Int} \times \operatorname{Int} \mid i_{1} \leq i_{2}\right\} \cup\left\{\mathbf{1}_{I}, \top\right\}
$$

and a binary operator $\oplus_{I}$ defined by:

$$
\begin{aligned}
\mathbf{1}_{I} \oplus_{I} x=x \oplus_{I} \mathbf{1}_{I}=x & \text { for all } x \in \mathbb{S}_{I} \\
\top \oplus_{I} x=x \oplus_{I} \top & =\top \text { for all } x \in \mathbb{S}_{I} \\
\left(i_{1}, i_{2}\right) \oplus_{I}\left(i_{3}, i_{4}\right) & = \begin{cases}\left(i_{1}, i_{4}\right) & \text { if } i_{2}+1=i_{3} \\
\top & \text { otherwise }\end{cases}
\end{aligned}
$$

It is easily checked that $I$ defines a monoid with identity $\mathbf{1}_{I}$.

The interval monoid abstractly describes summations of elements of in with respect to an arbitrary monoid, say, $M$. A pair $\left(i_{1}, i_{2}\right)$ abstractly describes the summation $\bigoplus_{M, i_{1} \leq i \leq i_{2}}$ in $[i]$. The identity $\mathbf{1}_{I}$ represents an empty interval of summations, thus is an abstraction of $\mathbf{1}_{M}$. Finally, the element $T$ represents all nonempty summations of elements in with respect to $\oplus_{M}$ that are not known to correspond to contiguous summations.

The $\oplus_{I}$ operator precisely captures the effect of summing two contiguous summations. For $i_{1} \leq i_{2}<i_{3}$,

$$
\begin{aligned}
& \bigoplus_{M, i_{1} \leq i \leq i_{2}} \operatorname{in}[i] \bigoplus_{M} \bigoplus_{M, i_{2}+1 \leq i \leq i_{3}} \operatorname{in}[i] \\
&=\bigoplus_{M, i_{1} \leq i \leq i_{3}} \operatorname{in}[i]
\end{aligned}
$$

and, correspondingly, $\left(i_{1}, i_{2}\right) \oplus_{I}\left(i_{2}+1, i_{3}\right)=\left(i_{1}, i_{3}\right)$. The way $\oplus_{I}$ treats $\mathbf{1}_{I}$ expresses the fact that adding an empty summation to either side of a summation interval has no effect. Finally, the treatment of $T$ by the operator captures the argument above: using only the properties of a monoid, it is not possible to transform a noncontiguous summation into a contiguous one by applying the $\oplus_{M}$ operator. Notice that $T$ is an absorbing element, or annihilating element, of $I$ : once a summation has become non-contiguous there can be no return.

Superficially, it may appear that the interval monoid is similar to the abstract domain of intervals that is commonly used in abstract interpretation [11]. The domains are in fact very different: an element $(i, j)$ of our interval of summations domain represents a single concrete value that can be expressed as a summation, whereas an element $[i, j]$ of the traditional domain of intervals represents the set of values $\{i, i+1, \ldots, j\}$.

We now define a condition on initial program stores which, for a given natural number $n$, ensures that the first $n$ elements of in are abstracted in the interval monoid by appropriate singleton summation intervals, and that all other array elements and variables of type $\mathbb{S}_{I}$ have values that are not known to be summation intervals.

Definition 4.4 (Singleton condition for sequential programs). Let $P$ be a generic program with in of type $\operatorname{Array}\left(\mathbb{S}_{X}\right)$. An initial state of $P[I]$ with variable store $\sigma_{\mathrm{v}}$ and array store $\sigma_{\mathrm{A}}$ satisfies the singleton condition for $n$ if

1. for all $v$ of type $\mathbb{S}_{X}$ in $P, \sigma_{\mathrm{v}}(v)=\top$;

2. for all $A$ of type Array $\left(\mathbb{S}_{X}\right)$ in $P$ and $k \in \operatorname{lnt}$,

$$
\sigma_{\mathrm{A}}(A)(k)= \begin{cases}(k, k) & \text { if } A=\text { in and } k \in\{0, \ldots, n-1\} \\ \top & \text { otherwise }\end{cases}
$$

We can now state our main theorem which shows that, with respect to generic prefix sums, the interval monoid is a sound and complete abstraction: a program implements a generic prefix sum 
if and only if it implements a prefix sum when instantiated with the interval monoid. In fact, our result is stronger than this: it shows that correctness of a generic prefix sum can be established by considering the interval monoid instantiation only for those initial stores that satisfy the singleton condition.

Theorem 4.5 (Soundness and completeness). Let $P$ be a generic program and $n$ a natural number. Then:

$P[I]$ computes an I-prefix sum of length $n$ for every initial state satisfying the singleton condition for $n$

\section{$P$ implements a generic prefix sum of length $n$.}

Given a generic program $P$, the main proof idea is to simulate the execution of $P[M]$ for any monoid $M$ by $P[I]$, and vice versa, whilst relating the states encountered in the executions. To this end, we first define a reification function.

Reification Let $M$ be a monoid and let ArrayStore denote the type of array stores. Define Reify ${ }_{M}: \mathbb{S}_{I} \times$ ArrayStore $\rightarrow \mathcal{P}\left(\mathbb{S}_{M}\right)$ :

$$
\begin{aligned}
\operatorname{Reify}_{M}\left(\left(i_{1}, i_{2}\right), \sigma_{\mathrm{A}}\right) & =\left\{\bigoplus_{M, i_{1} \leq i \leq i_{2}} \sigma_{\mathrm{A}}(\text { in })(i)\right\} \\
\operatorname{Reify}_{M}\left(\mathbf{1}_{I}, \sigma_{\mathrm{A}}\right) & =\left\{\mathbf{1}_{M}\right\} \\
\operatorname{Reify}_{M}\left(\top, \sigma_{\mathrm{A}}\right) & =\mathbb{S}_{M}
\end{aligned}
$$

Thus Reify ${ }_{M}$ maps a contiguous summation represented abstractly in the interval monoid to the set containing the corresponding concrete summation in the monoid $M$, and maps an unknown summation, represented by $T$ in the interval monoid, to the full set of elements of $M$.

Let $P$ be a generic program and for a monoid $M$ let State $_{P[M]}$ denote the set of all program states of $P[M]$. We lift Reify ${ }_{M}$ to map states of $P[I]$ to sets of states of $P[M]$. Formally,

$$
\text { Reify }_{M}: \text { State }_{P[I]} \rightarrow \mathcal{P}\left(\text { State }_{P[M]}\right)
$$

is defined by $\left(\sigma_{\mathrm{v}}^{\prime}, \sigma_{\mathrm{A}}^{\prime}, s s^{\prime}\right) \in \operatorname{Reify}_{M}\left(\sigma_{\mathrm{v}}, \sigma_{\mathrm{A}}, s s\right)$ if and only if

- for all $v$ of type $T$ in $P$

$$
\begin{array}{ll}
\sigma_{\mathrm{v}}^{\prime}(v)=\sigma_{\mathrm{v}}(v) & \text { if } T \neq \mathbb{S}_{X} \\
\sigma_{\mathrm{v}}^{\prime}(v) \in \operatorname{Reify}_{M}\left(\sigma_{\mathrm{v}}(v), \sigma_{\mathrm{A}}\right) & \text { if } T=\mathbb{S}_{X}
\end{array}
$$

- for all $A$ of type $\operatorname{Array}(T)$ in $P$ and $k \in \operatorname{lnt}$

$$
\begin{aligned}
& \sigma_{\mathrm{A}}^{\prime}(A)(k)=\sigma_{\mathrm{A}}(A)(k) \\
& \text { if } T \neq \mathbb{S}_{X} \\
& \sigma_{\mathrm{A}}^{\prime}(A)(k) \in \operatorname{Reify}_{M}\left(\sigma_{\mathrm{A}}(A)(k), \sigma_{\mathrm{A}}\right) \\
& \text { if } T=\mathbb{S}_{X}
\end{aligned}
$$

- there exists a generic program $Q$ such that $s s=Q[I]$ and $s s^{\prime}=Q[M]$.

Observe that the generic program $Q$ in the final condition is unique even if $I$ or $M$ is used in $P$, due to the discussion following Definition 4.1.

Simulation We can now prove our simulation result.

Lemma 4.6. Let $P$ be a generic program and $M$ a monoid. If $\mathcal{S}_{I}$ is a program state of $P[I]$ and $\mathcal{S}_{M} \in \operatorname{Reify}_{M}\left(\mathcal{S}_{I}\right)$ is a program state of $P[M]$, then

- if $\mathcal{S}_{I} \rightarrow_{\mathrm{S}} \mathcal{S}_{I}^{\prime}$, there exists a program state $\mathcal{S}_{M}^{\prime} \in \operatorname{Reify}_{M}\left(\mathcal{S}_{I}^{\prime}\right)$ of $P[M]$ such that $\mathcal{S}_{M} \rightarrow_{\mathrm{s}} \mathcal{S}_{M}^{\prime}$, and

- if $\mathcal{S}_{M} \rightarrow_{\mathrm{s}} \mathcal{S}_{M}^{\prime}$, there exists a program state $\mathcal{S}_{I}^{\prime}$ of $P[I]$ such that $\mathcal{S}_{I} \rightarrow_{\mathrm{s}} \mathcal{S}_{I}^{\prime}$ and $\mathcal{S}_{M}^{\prime} \in \operatorname{Reify}_{M}\left(\mathcal{S}_{I}^{\prime}\right)$.

Proof. Let $\mathcal{S}_{I}$ be a program state of $P[I]$ and $\mathcal{S}_{M} \in \operatorname{Reify}_{M}\left(\mathcal{S}_{I}\right)$ be a program state of $P[M]$. Moreover, let $Q$ be the generic program such that $Q[I]$ and $Q[M]$ are the program components of $\mathcal{S}_{I}$ and $\mathcal{S}_{M}$, respectively.
Observe that monoid substitution (Definition 4.1) satisfies the following algebraic laws for any monoid $M$ :

$$
\begin{aligned}
(v:=e)[M] & \equiv v:=(e[M]) \\
\left(A\left[e_{1}\right]:=e_{2}\right)[M] & \equiv A\left[e_{1}\right]:=\left(e_{2}[M]\right) \\
\left(\text { if }(e)\left\{s s_{1}\right\} \text { else }\left\{s s_{2}\right\}\right)[M] & \equiv \text { if }(e)\left\{s s_{1}[M]\right\} \text { else }\left\{s s_{2}[M]\right\} \\
(\text { while }(e)\{s s\})[M] & \equiv \text { while }(e)\{s s[M]\} \\
\varepsilon[M] & \equiv \varepsilon \\
(s ; s s)[M] & \equiv s[M] ; s s[M]
\end{aligned}
$$

where it is immediate by the typing rules and the definition of generic programs that neither $\oplus_{X}$ nor $\mathbf{1}_{X}$ can occur in either the expression $e_{1}$ of $A\left[e_{1}\right]:=e_{2}$ or the expression $e$ of a conditional or loop. For the same reason, it follows that if the first statement in $Q$ is a loop or conditional with guard $e$, then $e$ will evaluate identically in both $\mathcal{S}_{I}$ and $\mathcal{S}_{M}$. Thus, whatever the form of $Q$, the same rule from the operational semantics applies in both $\mathcal{S}_{I}$ and $\mathcal{S}_{M}$. Let the resulting program states after application of the rule be $\mathcal{S}_{I}^{\prime}$ and $\mathcal{S}_{M}^{\prime}$. By the algebraic laws it is now immediate that there exists a unique generic program $Q^{\prime}$ such that $Q^{\prime}[I]$ is the sequence of statements of $\mathcal{S}_{I}^{\prime}$ and $Q^{\prime}[M]$ is the sequence of statements of $\mathcal{S}_{M}^{\prime}$.

It remains to show that the conditions on the stores of $\mathcal{S}_{I}^{\prime}$ and $\mathcal{S}_{M}^{\prime}$ are satisfied. If the applied rule was one of S-ITE-T, S-ITEF, S-Loop-T, S-Loop-F, this is immediate, as the stores are identical before and after the steps.

For S-ASSIGN there are two cases to consider. If $v$ is not of type $\mathbb{S}_{X}$ in $Q$, it is immediate by the typing rules and the genericity of $Q$ that $e$ evaluates identically in both $\mathcal{S}_{I}$ and $\mathcal{S}_{M}$. If $v$ is of type $\mathbb{S}_{X}$ in $Q$, it follows by the typing rules and associativity of $\oplus_{X}$ that $e$ is an expression of the form $x_{0} \oplus_{X} \cdots \oplus_{X} x_{k}$ where for all $x_{i}$ with $0 \leq i \leq k$ either (a) the identity element $\mathbf{1}_{X}$, (b) a variable or (c) an array element. For each $x_{i}=A\left[e^{\prime}\right]$, the expression $e^{\prime}$ evaluates identically in both $\mathcal{S}_{I}$ and $\mathcal{S}_{M}$ by the typing rules and genericity of $Q$. The result is now immediate by the definition of $\oplus_{I}$ and the fact that (a) for each $x_{i}=\mathbf{1}_{X}$ we have $\mathbf{1}_{M} \in \operatorname{Reify}_{M}\left(\mathbf{1}_{I}, \sigma_{\mathrm{A}}\right)$, (b) for each variable $v$ among $x_{i}$ we have by assumption that $\sigma_{\mathrm{v}}^{\prime}(v) \in \operatorname{Reify}_{M}\left(\sigma_{\mathrm{v}}(v), \sigma_{\mathrm{A}}(\right.$ in $\left.)\right)$, and (c) for each $A\left[e^{\prime}\right]$ among $x_{i}$ we have $\sigma_{\mathrm{A}}^{\prime}(A)\left(\llbracket e^{\prime} \rrbracket_{\sigma_{\mathrm{A}}^{\prime}}^{\sigma_{\mathrm{A}}^{\prime}}\right) \in \operatorname{Reify}_{M}\left(\sigma_{\mathrm{A}}(A)\left(\llbracket e^{\prime} \rrbracket_{\sigma_{\mathrm{A}}}^{\sigma_{\mathrm{V}}}\right), \sigma_{\mathrm{A}}(\right.$ in $\left.)\right)$, where $\left(\sigma_{\mathrm{v}}, \sigma_{\mathrm{A}}\right)$ are the stores of $\mathcal{S}_{I}$ and $\left(\sigma_{\mathrm{v}}^{\prime}, \sigma_{\mathrm{A}}^{\prime}\right)$ those of $\mathcal{S}_{M}^{\prime}$.

For S-ARRAY observe that $e_{1}$ evaluates identically in both $\mathcal{S}_{I}$ and $\mathcal{S}_{M}$ by the typing rules and genericity of $Q$. Hence, the same array element is updated in each application of the rule. There are now two cases to consider, i.e., $A$ is or is not of type Array $\left(\mathbb{S}_{X}\right)$ in $Q$. These cases are identical to those of S-Assign.

Lemma 4.7. Let $P$ be a generic program, $M$ a monoid, and $n$ a natural number. If $\mathcal{S}_{M}$ is an initial state of $P[M]$, then there exists an initial state $\mathcal{S}_{I}$ of $P[I]$ satisfying the singleton condition for $n$ such that $\mathcal{S}_{M} \in \operatorname{Reify}_{M}\left(\mathcal{S}_{I}\right)$ and

- if $\mathcal{S}_{I} \rightarrow_{\mathrm{s}}^{*} \mathcal{S}_{I}^{\prime}$, there exists a program state $\mathcal{S}_{M}^{\prime} \in \operatorname{Reify}_{M}\left(\mathcal{S}_{I}^{\prime}\right)$ of $P[M]$ such that $\mathcal{S}_{M} \rightarrow_{\mathrm{s}}^{*} \mathcal{S}_{M}^{\prime}$, and

- if $\mathcal{S}_{M} \rightarrow_{\mathrm{s}}^{*} \mathcal{S}_{M}^{\prime}$, there exists a program state $\mathcal{S}_{I}^{\prime}$ of $P[I]$ such that $\mathcal{S}_{I} \rightarrow_{\mathrm{s}}^{*} \mathcal{S}_{I}^{\prime}$ and $\mathcal{S}_{M}^{\prime} \in \operatorname{Reify}_{M}\left(\mathcal{S}_{I}^{\prime}\right)$.

Proof. Let $\mathcal{S}_{M}$ be an initial state of $P[M]$. The lemma is immediate by induction on the number of steps applying Lemma 4.6 once we show that an initial state $\mathcal{S}_{I}$ of $P[I]$ exists that satisfies the singleton condition for $n$ such that $\mathcal{S}_{M} \in \operatorname{Reify}_{M}\left(\mathcal{S}_{I}\right)$. To this end, write $\mathcal{S}_{M}$ as $\left(\sigma_{\mathrm{v}}^{\prime}, \sigma_{\mathrm{A}}^{\prime}, P[M]\right)$ and define $\mathcal{S}_{I}$ as $\left(\sigma_{\mathrm{v}}, \sigma_{\mathrm{A}}, P[I]\right)$ with

- for all $v$ of type $T$ in $P$

$$
\sigma_{\mathrm{v}}(v)= \begin{cases}\sigma_{\mathrm{v}}^{\prime}(v) & \text { if } T \neq \mathbb{S}_{X} \\ \top & \text { if } T=\mathbb{S}_{X}\end{cases}
$$


- for all $A$ of type $\operatorname{Array}(T)$ in $P$ and $k \in \operatorname{lnt}$

$$
\sigma_{\mathrm{A}}(A)(k)= \begin{cases}(k, k) & \text { if } A=\text { in and } k \in\{0, \ldots, n-1\} \\ \top & \text { if } A=\text { in and } k \notin\{0, \ldots, n-1\} \\ \top & \text { if } A \neq \text { in and } T=\mathbb{S}_{X} \\ \sigma_{\mathrm{A}}^{\prime}(A)(k) & \text { otherwise }\end{cases}
$$

That $\mathcal{S}_{I}$ satisfies the singleton condition for $n$ and that we have $\mathcal{S}_{M} \in \operatorname{Reify}_{M}\left(\mathcal{S}_{I}\right)$ is now immediate.

Proof (Theorem 4.5). The $\Leftarrow$-direction is trivial, as $I$ is a monoid. For the $\Rightarrow$-direction, let $M$ be a monoid and $\mathcal{S}_{M}$ an initial state of $P[M]$. By Lemma 4.7 there exists an initial state $\mathcal{S}_{I}$ of $P[I]$ such that $\mathcal{S}_{I}$ satisfies the singleton condition and $\mathcal{S}_{M} \in \operatorname{Reify}\left(\mathcal{S}_{I}\right)$. All executions starting from $\mathcal{S}_{I}$ are terminating because $P[I] \mathrm{im}-$ plements an $I$-prefix sum. By Lemma 4.7, for every terminating execution (and thus every execution) $\mathcal{S}_{I} \rightarrow_{\mathrm{s}}^{*} \mathcal{S}_{I}^{\prime}$ there exists a corresponding terminating execution $\mathcal{S}_{M} \rightarrow_{\mathrm{s}}^{*} \mathcal{S}_{M}^{\prime}$ such that $\mathcal{S}_{M}^{\prime} \in \operatorname{Reify}_{M}\left(\mathcal{S}_{I}^{\prime}\right)$. Moreover, these are the only executions of $\mathcal{S}_{M}$ : if there would exist a non-terminating or another terminating execution starting from $\mathcal{S}_{M}$, then the induction argument from Lemma 4.7 would yield an additional execution starting from $\mathcal{S}_{I}$. Because $P[I]$ computes an $I$-prefix sum, we have for the array store $\sigma_{\mathrm{A}}$ of $\mathcal{S}_{I}^{\prime}$ that $\sigma_{\mathrm{A}}($ out $)(k)=\bigoplus_{I, 0 \leq i \leq k} \sigma_{\mathrm{A}}($ in $)(i)=$ $(0, k)$ for all $k \in\{0, \ldots, n-1\}$. Hence, by definition of reification for the array store $\sigma_{\mathrm{A}}^{\prime}$ of $\mathcal{S}_{M}^{\prime}$ we have that $\sigma_{\mathrm{A}}^{\prime}($ out $)(k)=$ $\bigoplus_{M, 0 \leq i \leq k} \sigma_{\mathrm{A}}^{\prime}($ in $)(i)$ for all $k \in\{0, \ldots, n-1\}$.

\section{Extension to Data-Parallel Programs}

We next consider data-parallel programs, or kernels, in which threads synchronise by means of barriers.

Syntax, typing and semantics We extend the language of Figure 4 with a barrier synchronisation statement as follows:

$$
\text { stmt } s::=\cdots \mid \text { barrier }
$$

The typing rule for barrier is straightforward:

$$
\overline{\Gamma \vdash \text { barrier : Unit }}(\text { T-BARRIER) }
$$

The typing rules for expressions and all other statements are as before (see Figure 5).

Given a finite set of thread identifiers $D \subset$ Int, a kernel (program) state is a tuple $\left(\sigma_{\mathrm{A}}, K\right)$ with $\sigma_{\mathrm{A}}$ an array store and $K$ a map from $D$ to (variable store, sequence of statements)-pairs such that for each $t \in D$ and variable store $\sigma_{\mathrm{v}}$ of $K(t)$ we have $\sigma_{\mathrm{v}}(t i d)=t$. The array store $\sigma_{\mathrm{A}}$ represents arrays that are shared among all threads, and $K$ represents the local variables and instruction sequence for each thread. The variable tid represents the identity of a thread, and must occur read-only in every program $P$. Our theoretical presentation does not depend on the existence of $t i d$, but the prefix sum implementations we evaluate in Section 7 rely on each thread having a unique identifier.

Figure 7 gives the operational semantics of our kernel programming language, where $\rightarrow_{\mathrm{s}}$ is as defined in Figure 6 . The semantics is a standard interleaving semantics (see rule K-STEP) with an additional rule for barrier synchronisation (rule K-BARRIER). Rule K-BARRIER checks whether all threads are either at a barrier or have terminated; the additional condition that at least one thread must be at a barrier ensures that rule K-BARRIER and termination are mutually exclusive.

The semantics assumes that statements are executed atomically, so that for example a thread can execute a shared state update such as $A[i]:=A[j]+1$ in a single step. This assumption is not valid in practice: such a statement would involve issuing separate (and possibly multiple) load and store instructions between which other threads could interleave. Even if we refined the semantics to reflect this, we would still need to account for the weak memory models of modern architectures. However, if a program is free from data races, which we define formally below, the effects of this assumption are not visible. The verification technique for GPU implementations of parallel prefix sums, which we present in Section 6, depends on proving that a GPU kernel is free from data races.

Our rules for barrier synchronisation follow the MPI programming model [14] in which it is valid for parallel processes to synchronise at syntactically distinct barriers. In GPU programming models, such as OpenCL and CUDA the rules for barrier synchronisation are stricter, requiring that all threads synchronise at the same barrier, and that if a barrier is inside a loop all threads must have executed the same number of loop iterations on reaching the barrier [19, 27]; precise semantics for barrier synchronisation in GPU kernels have been formally specified $[1,10]$. Our results for the looser barrier synchronisation model of MPI makes our technique more widely applicable. Adding stricter conditions for barrier synchronisation does not affect our theoretical results, and the GPUVerify tool used as part of our verification method checks the stricter conditions required in the GPU setting.

Given a kernel program $P$, an initial state of $P$ is any kernel state $\left(\sigma_{\mathrm{A}}, K\right)$ where, for every thread $t$, the sequence of statements of $K(t)$ is $P$. An execution of a program $P$ starts from an initial state. Maximal executions and termination for an initial state are defined as for sequential programs. Note that the interleaving nature of the semantics means that there may be multiple maximal executions, contrary to the sequential case.

Prefix sums and generic prefix sums Computation and implementation of a prefix sum is defined as in the sequential case (see Definition 3.1) with $P$ interpreted as a kernel program. A generic kernel program and generic prefix sum are also defined as in the sequential case (Definition 4.1).

Interval monoid Employing the interval monoid as is (Definition 4.3), we extend the definition of the singleton condition to kernel programs, taking into account that there is now a variable store per thread.

Definition 5.1 (Singleton condition for kernel programs). Let $P$ be a generic program with in of type $\operatorname{Array}(X)$. An initial state $\left(\sigma_{\mathrm{A}}, K\right)$ of $P[I]$ satisfies the singleton condition for $n$ if

1. for all $t \in D$ and $v$ of type $\mathbb{S}_{X}$ in $P, \sigma_{\mathrm{v}}(v)=\top$ with $\sigma_{\mathrm{v}}$ the variable store of $K(t)$;

2. condition 2 of Definition 4.4 is satisfied

Soundness and completeness Our soundness and completeness theorem for sequential programs, Theorem 4.5, can now be stated identically for kernel programs. The proof strategy is the same, using reification of abstract states to concrete states to set up a simulation, using lemmas analogous to Lemma 4.6 and Lemma 4.7. We explain the notable differences in how the proof is set up.

The Reify ${ }_{M}$ function is adapted to map states of kernel programs $P[I]$ to sets of states of kernel programs $P[M]$. The function treats the array store as before, but must take into account the fact that each thread now has its own variable store and program. Thus, while reification of array stores still operates at the level of the whole program state, reification of the variable store and program component now operates at the level of each individual thread. The definition is left unchanged otherwise.

The proof of Lemma 4.6 translates to the case of kernel programs by observing that

$$
\text { barrier }[M] \equiv \text { barrier }
$$




$$
\begin{aligned}
& \frac{K(t)=\left(\sigma_{\mathrm{v}}, s s\right) \quad\left(\sigma_{\mathrm{v}}, \sigma_{\mathrm{A}}, s s\right) \rightarrow_{\mathrm{s}}\left(\sigma_{\mathrm{v}}^{\prime}, \sigma_{\mathrm{A}}^{\prime}, s s^{\prime}\right) \quad K^{\prime}=K\left[t \mapsto\left(\sigma_{\mathrm{v}}^{\prime}, s s^{\prime}\right)\right]}{\left(\sigma_{\mathrm{A}}, K\right) \rightarrow_{\mathrm{k}}\left(\sigma_{\mathrm{A}}^{\prime}, K^{\prime}\right)}(\mathrm{K}-\mathrm{STEP})
\end{aligned}
$$

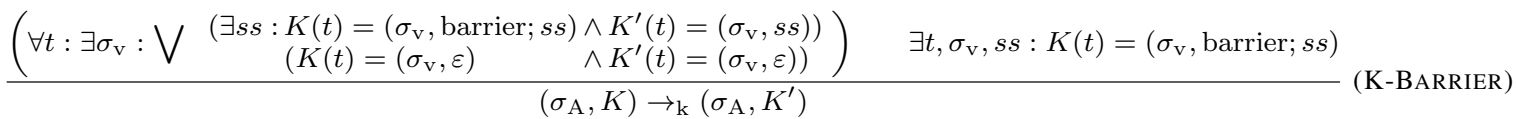

Figure 7. Operational semantics of our kernel programming language, extending the sequential rules of Figure 6

and that by this algebraic law and the algebraic laws from the proof of Lemma 4.6 we have that the same rule will be applied in both the case of $\mathcal{S}_{I}$ and $\mathcal{S}_{M}$, where the proof of Lemma 4.6 is embedded in the treatment of the K-STEP case.

In proving Lemma 4.7 for kernel programs, we must relate an initial state $\mathcal{S}_{M}=\left(\sigma_{\mathrm{A}}^{\prime}, K^{\prime}\right)$ of $P[M]$ to an initial state $\mathcal{S}_{I}$ of $P[I]$ that satisfies the singleton condition. Otherwise, the proof is identical to that of Lemma 4.7. The definition of the initial state $\mathcal{S}_{I}$ is easily adapted from the definition in the proof of Lemma 4.7 by applying the definition for the variable store to the variable store of each individual thread; the definition is left unchanged otherwise.

Data race freedom We now define what it means for a kernel program to exhibit a data race. We show that if a kernel program is data race-free then, due to the properties of barrier synchronisation, the program computes a deterministic result. The verification technique we present in Section 6 depends on this guarantee of determinism and, as discussed above, establishing race-freedom of a kernel avoids the need to reason about weak memory semantics or the granularity of memory accesses.

Say that we read from an array element $A[i]$ in an execution step if $\sigma_{\mathrm{A}}(A)(i)$ is referenced during the evaluation of any of the expressions occurring in the step. Likewise, say that we write to an array element $A[i]$ in an execution step if $\sigma_{\mathrm{A}}(A)(i)$ is updated in the step. An array element $A[i]$ is accessed if it is either read from or written to in the execution step. Observe that an array element can be accessed multiple times in a single execution step, e.g., when evaluating $A[t i d]:=A[t i d] \oplus_{M} \mathbf{1}_{M}$.

Definition 5.2. Let $\mathcal{S}_{0} \rightarrow_{\mathrm{k}}^{*} \mathcal{S}_{n}$ be an execution of a kernel program $P$. The execution is said to have a data race if there are steps $\mathcal{S}_{i} \rightarrow_{\mathrm{k}} \mathcal{S}_{i+1}$ and $\mathcal{S}_{j} \rightarrow_{\mathrm{k}} \mathcal{S}_{j+1}$ along the execution such that:

- distinct threads are responsible for these steps,

- a common array element is accessed in both execution steps,

- at least one of the accesses writes to the array element,

- no application of K-BARRIER occurs in between the accesses.

A program $P$ is data race-free if for every initial state $\mathcal{S}_{0}$ of $P$ and execution starting from $\mathcal{S}_{0}$ it holds that the execution does not have a data race.

Theorem 5.3. Let $P$ be a generic kernel program and let $M$ be a monoid. Then: $P[M]$ is data race-free $\Longleftrightarrow P\left[M^{\prime}\right]$ is data racefree for all monoids $M^{\prime}$.

Proof. The $\Leftarrow$-direction is trivial, as $M$ is a monoid. For the $\Rightarrow$ direction, observe that neither the control-flow nor the array accesses can be influenced by the choice of $M^{\prime}$ by the typing rules and genericity of $P$.

We now argue that data race-free kernel programs behave deterministically. Say that a barrier synchronisation occurs during execution of a program whenever the K-BARRIER rule of Figure 7 applies. Suppose that a kernel program is race-free, and consider executing the program from an initial state $\mathcal{S}$. Race-freedom means that the execution of one thread cannot depend upon the actions of another until all threads participate in a barrier synchronisation. Thus while the threads may interleave nondeterminstically, their individual execution is deterministic and when the first barrier synchronisation occurs (assuming all threads reach a barrier without diverging), the program state $\mathcal{S}_{1}$ is deterministic. By a similar argument, individual thread execution until the next barrier synchronisation is deterministic, leading to a deterministic program state $\mathcal{S}_{2}$ when the second barrier synchronisation occurs. Applying this argument and using induction on program executions, we can prove the following:

Lemma 5.4. Let $P$ be a data race-free kernel program and let $\mathcal{S}$ be an initial state of $P$. If there exists a finite, maximal execution starting from $\mathcal{S}$ with final array store $\sigma_{\mathrm{A}}$, then all executions starting from $\mathcal{S}$ are finite and for all maximal executions the final array store is $\sigma_{\mathrm{A}}$.

\section{An Automated Verification Technique}

Our theoretical results show that to verify functional correctness of a generic parallel prefix sum of length $n$, implemented as a barriersynchronising program, it suffices to prove that the program is free from data races and then test that the program behaves correctly with respect to the interval monoid for every initial state with in $=[(0,0),(1,1), \ldots,(n-1, n-1)]$. In practice, a library routine for computing a prefix sum takes no input except for in so that there is just a single input to test.

GPU kernels are barrier synchronising programs that are required to be data race-free: the semantics of an OpenCL or CUDA kernel is undefined if the kernel exhibits data races [19, 27]. Thus our results suggest the following method for verifying functional correctness of GPU kernels that claim to implement generic prefix sums with respect to a single input array:

\section{Prove that the GPU kernel is data race-free.}

2. Run the interval monoid test to check functional correctness.

Steps 1 and 2 can be conducted independently, but the functional correctness guarantee provided by step 2 is conditional on the result of step 1. We now discuss how we have implemented a practical verification method for generic prefix sums written in OpenCL, based on this approach.

Representing a generic prefix sum The OpenCL programming model does not support generic functions directly. However, it is easy to describe a generic prefix sum by writing a kernel that uses a symbol TYPE and a macro OPERATOR as placeholders for the concrete type and operator with respect to which the prefix sum should be executed. A concrete choice of type and operator can be chosen by including a header file that specifies TYPE and OPERATOR.

Race analysis Step 1 above can be discharged to any sound verifier for GPU kernels capable of proving race-freedom. For GPU kernels this includes the PUG [24] and GPUVerify [1] tools, which rely on SMT solvers, and the tool described in [23] which performs verification using a combination of dynamic and static analysis. In 
our experiments (Section 7) we use the GPUVerify tool because it is actively maintained and publicly available, and is the only tool supporting the multi-platform OpenCL programming model, which allows us to evaluate step 2 above on a number of platforms.

By Theorem 5.3, we can prove race-freedom of a generic prefix sum using any choice of TYPE and OPERATOR. In our experiments we use the interval monoid.

Running the interval monoid test case Step 2 above requires an encoding of the interval monoid. Observe that to check a prefix sum of length $n$ we need only encode elements $(i, j)$ of the interval monoid for $0 \leq i \leq j<n$, as well as the $\mathbf{1}_{I}$ and $\top$ elements. An element can thus be encoded using $O(\lg (n))$ bits, meaning that $O(n \lg (n))$ space is required for the test input and result. With this encoding, the interval monoid test case can be executed as a regular OpenCL kernel.

Soundness, completeness and automation Our verification strategy is sound due to the guarantees provided by the version of Theorem 4.5 for kernel programs, together with Theorem 5.3 and Lemma 5.4, and the requirement that a sound method for verifying race-freedom is used. As with any dynamic technique, soundness of the testing phase of our approach depends on the probity of the compiler, driver and hardware implementation of the architecture on which the test is executed. In our experiments we guard against this source of unsoundness by testing with respect to multiple, diverse platforms.

Our verification strategy is only complete if the technique used to prove race freedom is complete. The GPUVerify method which we employ is not complete: the technique uses both thread abstraction and loop invariant abstraction, which can lead to false positive data race reports.

A small amount of manual effort is required in order to prove race-freedom of prefix sum kernels: GPUVerify tries to perform automatic inference of invariants required to prove race-freedom, but often this inference is not strong enough and manual invariants must be supplied by the programmer. However, these invariants are checked by GPUVerify - they are not taken on trust by the tool. In the examples we consider in Section 7 we disabled automatic invariant inference and supplied a small number of relatively simple, non-quantified loop invariants by hand. The dynamic analysis phase of our verification method is fully automatic.

Handling extended programming language features Our sequential language and its data-parallel extension omit real-world language features such as procedures, unstructured control flow, and pointers (with associated aliasing issues). We are confident that our results apply directly in this extended setting under the condition that data of generic type $\mathbb{S}_{X}$ are never accessed via pointers with different element types. This is important for the testing part of our approach; without this restriction it would be possible to pass our test case by casting the out array to a char pointer and writing the desired final result for the interval monoid byte-by-byte. Proving race-freedom in the presence of intricate pointer manipulation can be challenging, but is possible with the right invariants and is supported by GPUVerify. Because GPUVerify operates at the level of control flow graphs using the techniques of [10], unstructured control flow is also supported.

Nevertheless, the prefix sum implementations we have seen in practice do not manipulate pointers in complex ways, call auxiliary procedures or exhibit unstructured control flow.

As discussed in Section 3, the requirement that the input array in be read-only is for ease of presentation only and can be dropped in practice, allowing our technique to be used in verifying prefix sums that operate in place.

\begin{tabular}{lccc} 
Algorithm & Depth & Size & Fanout \\
\hline Sequential & $n$ & $n$ & 2 \\
Kogge-Stone & $\lg n$ & $n \lg n-(n-1)$ & 2 \\
Sklansky & $\lg n$ & $(n / 2) \lg n$ & $n$ \\
Brent-Kung & $(2 \lg n)-1$ & $2 n-\lg n-2$ & 2 \\
Blelloch & $2 \lg n$ & $2(n-1)$ & 2 \\
\hline
\end{tabular}

Table 2. Characteristics of the prefix sum algorithms considered in this paper where $n$ is the number of elements

\section{Experimental Evaluation}

We demonstrate the effectiveness of our method for prefix sum verification in practice by applying it to four different algorithms implemented as OpenCL kernels.

All materials required to reproduce our experimental results, including implementations all kernels, are available online. ${ }^{5}$

Details of prefix sum kernels We evaluate our technique using four different, well-known prefix sum algorithms: KoggeStone [21], Sklansky [34], Brent-Kung [5] and Blelloch [4]. The Blelloch algorithm is an exclusive prefix sum.

Figure 8 provides a circuit diagram description for each algorithm. There is a wire for each input and data flows top-down through the circuit. Each node • performs the binary associative operator on its two inputs and produces an output that passes downward and also optionally across the circuit (through a diagonal wire). Table 2 gives a comparison of these algorithms, as well as the straightforward sequential implementation, in terms of circuit characteristics: depth, the number of levels; size, the number of nodes; and fanout, the maximum number of outbound wires per node, as the size of the input (or width) $n$ of the circuit varies. The depth of a circuit indicates how long it takes for the result to propagate through the circuit, and thus dictates the time taken for prefix sum computation. Size indicates how many gates would be required to build the circuit in hardware, while fanout indicates the required driving capacitance of the output gate. A software implementation of a prefix sum is work efficient if the number of operations required is within the same order of magnitude as the sequential version. The size column of Figure 2 indicates that Blelloch and Brent-Kung are work-efficient, while Kogge-Stone and Sklansky are not. A more detailed discussion of these characteristics can be found in [16].

Through a survey of several GPU code repositories (the AMD APP SDK, ${ }^{6}$ the NVIDIA CUDA SDK, ${ }^{7}$ and the SHOC [12], Rodinia [7] and Parboil [37] benchmarks) we found Kogge-Stone to be the most widely used GPU implementation of a prefix sum in practice, Blelloch to be used where an exclusive prefix sum is required, and Brent-Kung employed several times in one large CUDA kernel that computes eigenvalues of a matrix. Sklansky is the oldest prefix sum algorithm we consider; we did not see it used in practical GPU kernels.

Experimental setup As discussed in Section 6, we use the GPUVerify tool [1] to prove that each kernel is free from data races. These experiments were performed on a Linux machine with a 1.15 GHz AMD Phenom 9600B 4 core processor, using a version of GPUVerify downloaded from the tool web page ${ }^{8}$ on 26 June 2013.

We then verify functional correctness for each kernel by running the interval of summations test case. We give results for five different platforms: two NVIDIA GPUs - a $1.16 \mathrm{GHz}$ GeForce GTX 570

\footnotetext{
$\overline{5}$ http://multicore.doc.ic.ac.uk/tools/GPUVerify/POPL14

${ }^{6}$ http://developer.amd.com/tools/heterogeneous-computing/ amd-accelerated-parallel-processing-app-sdk/

${ }^{7}$ https://developer.nvidia.com/gpu-computing-sdk

${ }^{8}$ http://multicore.doc.ic.ac.uk/tools/GPUVerify/
} 


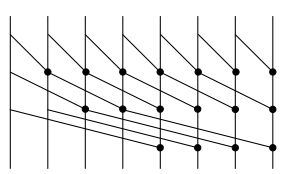

(a) Kogge-Stone

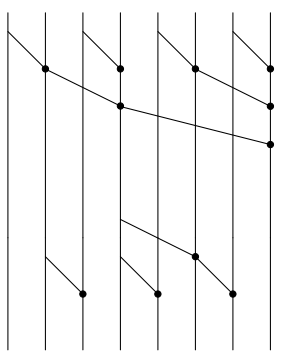

(c) Brent-Kung

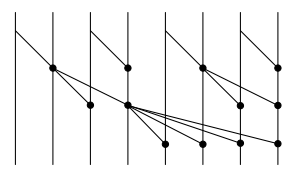

(b) Sklansky

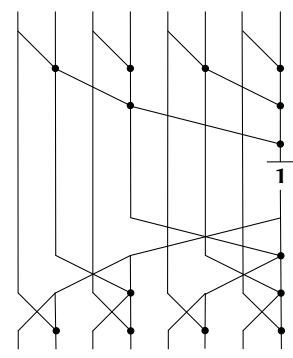

(d) Blelloch
Figure 8. Circuit representations of the prefix sum algorithms for $n=8$ elements

and a $1.15 \mathrm{GHz}$ Tesla M2050; two Intel CPUs - a $2.13 \mathrm{GHz} 4$ core Xeon E5606 and a $2.67 \mathrm{GHz} 6$ core Xeon X5650; and one ARM GPU - a $533 \mathrm{MHz} 4$ core Mali T604. These devices exhibit a range of power and performance characteristics. The NVIDIA GPUs offer a large number of parallel processing elements running at a relatively low clock-rate; the Intel CPUs run at a higher clock-rate but exhibit less parallelism; and the ARM GPU is designed for high power-efficiency. We chose to run on multiple platforms to guard against possible unsound results as a result of a particular compiler, driver or hardware configuration. Each experiment was run with a timeout of 1 hour. All the timing results we present are averages over three runs.

In [38], Voigtländer shows for sequential programs that a generic prefix sum is correct for all input lengths if it can be shown to behave correctly for all input lengths with respect to two binary operators over a set of three elements. He shows further that it is sufficient to consider $O\left(n^{2}\right)$ test inputs: $n(n+1) / 2$ tests using the first operator and $n-1$ tests using the second operator. The result of [38] considers all possible $n$, but also restricts so that a prefix sum of a specific length $n$ can be shown correct by testing the corresponding set of inputs. Our Lemma 5.4 allows us to lift this method to race-free barrier-synchronising programs. Thus, by way of comparison, we also tried dynamically verifying the prefix sum kernels by running, for each element size, the set of Voigtländer tests. We discuss Voigtländer's paper further in the related work (Section 8).

Verification using the interval of summations test case and the Voigtländer tests both require race-freedom to be established, thus the overhead of race checking applies to both approaches.

Results for proving race-freedom Table 3 presents verification times, in seconds, for proving race-freedom of each of our prefix sum kernels using GPUVerify, for a selection of power-of-two element counts up to $2^{31}$. Times for the power-of-two thread counts not shown are in the same order of magnitude.

The results show that verification time is more-or-less independent of the number of threads executing the kernel. This is because GPUVerify checks race-freedom for a kernel with respect to an arbitrary pair of threads, and reasons about loops using invariants rather than by unrolling. This excellent scalability demonstrates that the data race analysis aspect of our verification method is practical for very large arrays.

As noted in Section 6, we disabled automatic invariant inference in GPUVerify as it did not allow fully automatic verification of our examples. Instead we provided relatively simple, non-quantified loop invariants for each of our kernels in order for verification to succeed. For example, for the loop of the Kogge-Stone kernel shown in Figure 2 we provided the following invariant (no further invariants were necessary):

$$
\text { no_read (out) } \wedge \text { no_write(out) }
$$

which specifies that no access to the out array is in-flight at the head of the loop. The most intricate invariant we had to specify, for the Blelloch kernel, was:

$(\mathrm{d} \&(\mathrm{~d}-1))=0 \wedge(($ offset $=0 \wedge \mathrm{d}=N) \vee \mathrm{d} *$ offset $=N)$.

Here, $d$ and offset are loop counters, with $d$ increasing in powersof-two from 1 to $N$, and offset decreasing in powers-of-two from $N$ to 1 , and finally reaching zero. The conjunct $(\mathrm{d} \&(\mathrm{~d}-1))=0$ uses the bitwise-and operator, $\&$, to compactly express that $d$ must be a power-of-two or zero.

Counting each top-level conjunct of a loop invariant as a separate invariant, the number of invariants required for verification of each kernel was as follows: (Kogge-Stone, 2); (Sklansky, 1); (Brent-Kung, 4); (Blelloch, 6). The invariants did not have to be tailored for individual thread counts.

Results for dynamic analysis The I rows of Table 4 present, for each prefix sum and platform, the time in seconds taken to run the single interval of summations test for power-of-two array sizes from $2^{9}$ to $2^{14}$ and $2^{18}$ to $2^{20}$ (results in between follow a similar trend). For each array size we also show in the \#Voigtländer tests row the total number of tests that would need to be run to verify the kernel using Voigtländer's method and the $\mathbf{V}$ rows show how long these tests took to run. The times are end-toend, taking into account device initialisation, creation of memory buffers, compilation of the OpenCL kernel, ${ }^{9}$ copying data into memory buffers, running the test and validating the result. For the Voigtländer tests we used a wrapper so that device initialisation, creation of memory buffers and kernel compilation is performed only once for each array size. We note that while in theory an array of length $n$ can be processed by $n / 2$ concurrent threads (or $n$ threads in the case of Kogge-Stone), it is up to the OpenCL runtime to decide how to schedule threads across hardware resources, and that in practice a large number of threads will be launched in a series of waves.

From the I rows it can be seen that running the interval of summations test case is very fast on all platforms, even for arrays of length $2^{20}$. It may seem surprising that the runtime does not increase significantly as the array size increases. This is because (a) computing with the interval monoid is very cheap, requiring only integer equality testing and addition by a constant and (b) the expense of online compilation of the OpenCL kernel, which dominates the runtime of these simple tests.

The $\mathbf{V}$ rows show that, unsurprisingly, running a quadratic number of tests per array size does not scale well. On all platforms, we found that sizes of $n \geq 2^{14}$ exhausted our time limit of 1 hour, showing that the Voigtländer method will not scale to large arrays.

\section{Related Work}

Relationship to results by Voigtländer and Sheeran The closest work to this paper is work by Voigtländer [38] which proves two interesting results for sequential generic prefix sums.

\footnotetext{
${ }^{9}$ In the OpenCL model, a kernel is compiled at runtime using an online compiler, so that it need not be pre-compiled for any given device [19].
} 


\begin{tabular}{|c|c|c|c|c|c|c|c|c|c|c|c|c|c|c|c|}
\hline$n$ & $2^{1}$ & $2^{2}$ & $2^{3}$ & $\ldots$ & $2^{8}$ & $2^{9}$ & $2^{10}$ & $2^{11}$ & $2^{12}$ & $2^{13}$ & $2^{14}$ & $\ldots$ & $2^{29}$ & $2^{30}$ & $2^{31}$ \\
\hline Kogge-Stone & 5.7 & 5.8 & 5.9 & & 5.8 & 5.8 & 6.0 & 5.6 & 5.8 & 5.7 & 5.6 & & 6.4 & 6.4 & 6.6 \\
\hline Sklansky & 5.6 & 6.1 & 6.5 & & 6.8 & 6.6 & 6.7 & 6.8 & 6.7 & 6.5 & 6.4 & & 7.3 & 8.1 & 7.9 \\
\hline Brent-Kung & 5.9 & 6.7 & 7.4 & & 12.3 & 11.4 & 12.9 & 10.2 & 10.3 & 10.7 & 12.6 & & 14.9 & 11.1 & 16.4 \\
\hline Blelloch & 6.0 & 7.4 & 9.0 & & 12.4 & 12.6 & 13.8 & 12.0 & 14.1 & 11.6 & 12.3 & & 15.5 & 13.0 & 14.8 \\
\hline
\end{tabular}

Table 3. Time (in seconds) taken to prove race freedom for prefix sum kernels, for increasing array lengths. An array of length $n$ is processed by $n / 2$ threads, except in the case of Kogge-Stone where $n$ threads are used.

\begin{tabular}{|c|c|c|c|c|c|c|c|c|c|c|c|}
\hline \multicolumn{2}{|c|}{$\begin{array}{c}n \\
\text { \#Voigtländer tests }\end{array}$} & $\begin{array}{r}2^{9} \\
131,839\end{array}$ & $\begin{array}{r}2^{10} \\
525,823\end{array}$ & $\begin{array}{r}2^{11} \\
2.1 \times 10^{6}\end{array}$ & $\begin{array}{r}2^{12} \\
8.4 \times 10^{6}\end{array}$ & $\begin{array}{r}2^{13} \\
3.4 \times 10^{7}\end{array}$ & $\begin{array}{r}2^{14} \\
1.3 \times 10^{8}\end{array}$ & $\cdots$ & $\begin{array}{r}2^{18} \\
3.4 \times 10^{10}\end{array}$ & $\begin{array}{r}2^{19} \\
1.4 \times 10^{11}\end{array}$ & $\begin{array}{r}2^{20} \\
5.5 \times 10^{11}\end{array}$ \\
\hline \multicolumn{12}{|c|}{ NVIDIA GeForce GTX 570} \\
\hline \multirow[t]{2}{*}{ Kogge-Stone } & $\mathbf{V}$ & 19.2 & 76.2 & 337.6 & 1463.9 & - & - & & - & - & - \\
\hline & I & 0.4 & 0.5 & 0.3 & 0.4 & 0.3 & 0.4 & & 0.4 & 0.4 & 0.4 \\
\hline \multirow[t]{2}{*}{ Sklansky } & $\mathbf{V}$ & 18.5 & 71.8 & 320.2 & 1438.3 & - & - & & - & - & - \\
\hline & I & 0.4 & 0.4 & 0.4 & 0.4 & 0.4 & 0.4 & & 0.4 & 0.4 & 0.4 \\
\hline \multirow[t]{2}{*}{ Brent-Kung } & $\mathbf{V}$ & 19.0 & 69.3 & 317.3 & 1454.3 & - & - & & - & - & - \\
\hline & I & 0.5 & 0.4 & 0.4 & 0.4 & 0.4 & 0.4 & & 0.4 & 0.4 & 0.4 \\
\hline \multirow{2}{*}{ Blelloch } & V & 19.2 & 75.4 & 324.2 & 1595.3 & - & - & & - & - & - \\
\hline & $\mathbf{I}$ & 0.5 & 0.4 & 0.4 & 0.4 & 0.4 & 0.4 & & 0.4 & 0.4 & 0.4 \\
\hline \multicolumn{12}{|c|}{ NVIDIA Tesla M2050 } \\
\hline \multirow[t]{2}{*}{ Kogge-Stone } & $\mathbf{V}$ & 14.6 & 36.1 & 160.9 & 653.0 & 2796.8 & - & & - & - & - \\
\hline & I & 0.3 & 0.3 & 0.6 & 0.5 & 0.5 & 0.5 & & 0.6 & 0.5 & 0.6 \\
\hline \multirow[t]{2}{*}{ Sklansky } & $\mathbf{V}$ & 12.2 & 32.5 & 149.6 & 609.3 & 2601.5 & - & & - & - & - \\
\hline & I & 0.3 & 0.3 & 0.5 & 0.5 & 0.5 & 0.5 & & 0.6 & 0.6 & 0.6 \\
\hline \multirow{2}{*}{ Brent-Kung } & $\mathbf{V}$ & 10.9 & 35.9 & 165.7 & 678.1 & 2889.2 & - & & - & - & - \\
\hline & I & 0.3 & 0.3 & 0.6 & 0.5 & 0.6 & 0.5 & & 0.6 & 0.6 & 0.6 \\
\hline \multirow{2}{*}{ Blelloch } & V & 10.9 & 36.7 & 166.0 & 679.9 & 2931.8 & - & & - & - & - \\
\hline & $\mathbf{I}$ & 0.3 & 0.3 & 0.5 & 0.6 & 0.6 & 0.6 & & 0.6 & 0.6 & 0.6 \\
\hline \multicolumn{12}{|c|}{ Intel Xeon X5650 } \\
\hline \multirow[t]{2}{*}{ Kogge-Stone } & $\mathbf{V}$ & 21.1 & 109.8 & 660.8 & 3467.1 & - & - & & - & - & - \\
\hline & I & 1.3 & 1.3 & 1.3 & 1.3 & 1.3 & 1.3 & & 1.3 & 1.3 & 1.3 \\
\hline \multirow{2}{*}{ Sklansky } & $\mathbf{V}$ & 12.2 & 78.4 & 404.8 & 2003.9 & - & - & & - & - & - \\
\hline & I & 1.3 & 1.3 & 1.3 & 1.3 & 1.3 & 1.3 & & 1.3 & 1.3 & 1.3 \\
\hline \multirow[t]{2}{*}{ Brent-Kung } & V & 12.6 & 80.4 & 469.9 & 2272.5 & - & - & & - & - & - \\
\hline & I & 1.3 & 1.3 & 1.3 & 1.3 & 1.3 & 1.3 & & 1.3 & 1.3 & 1.3 \\
\hline \multirow[t]{2}{*}{ Blelloch } & $\mathbf{V}$ & 12.9 & 80.7 & 472.1 & 2332.5 & - & - & & - & - & - \\
\hline & I & 1.3 & 1.3 & 1.3 & 1.3 & 1.3 & 1.3 & & 1.3 & 1.4 & 1.3 \\
\hline \multicolumn{12}{|c|}{ Intel Xeon E5606 } \\
\hline \multirow[t]{2}{*}{ Kogge-Stone } & V & 107.0 & 909.2 & - & - & - & - & & - & - & - \\
\hline & I & 1.9 & 1.9 & 2.1 & 2.1 & 2.0 & 2.1 & & 2.4 & 2.7 & 3.2 \\
\hline \multirow[t]{2}{*}{ Sklansky } & V & 38.7 & 266.0 & 1221.5 & - & - & - & & - & - & - \\
\hline & I & 1.9 & 1.9 & 2.0 & 2.1 & 2.0 & 2.0 & & 2.2 & 2.3 & 2.4 \\
\hline \multirow[t]{2}{*}{ Brent-Kung } & V & 51.5 & 409.1 & 1793.8 & - & - & - & & - & - & - \\
\hline & I & 2.0 & 2.0 & 2.1 & 2.1 & 2.1 & 2.1 & & 2.3 & 2.3 & 2.5 \\
\hline Blelloch & $\mathbf{V}$ & 54.3 & 429.9 & 1900.3 & - & - & - & & - & - & - \\
\hline & I & 1.9 & 2.0 & 2.1 & 2.1 & 2.1 & 2.1 & & 2.4 & 2.4 & 2.6 \\
\hline & & & & & $\mathbf{A R I}$ & Mali T604 & & & & & \\
\hline Kogge-Stone & $\mathbf{V}$ & 287.0 & 1166.1 & - & - & - & - & & - & - & - \\
\hline & I & 0.3 & 0.3 & 0.3 & 0.3 & 0.3 & 0.3 & & 0.4 & 0.4 & 0.5 \\
\hline Sklansky & $\mathbf{V}$ & 287.2 & 1147.6 & - & - & - & - & & - & - & - \\
\hline & I & 0.3 & 0.3 & 0.3 & 0.3 & 0.3 & 0.3 & & 0.4 & 0.4 & 0.5 \\
\hline Brent-Kung & $\mathbf{V}$ & 287.4 & 1108.1 & - & - & - & - & & - & - & - \\
\hline & I & 0.4 & 0.4 & 0.4 & 0.4 & 0.4 & 0.4 & & 0.4 & 0.5 & 0.6 \\
\hline Blelloch & $\mathbf{V}$ & 277.0 & 1105.3 & - & - & - & - & & - & - & - \\
\hline & I & 0.4 & 0.4 & 0.4 & 0.4 & 0.4 & 0.4 & & 0.4 & 0.5 & 0.6 \\
\hline
\end{tabular}

Table 4. Time (in seconds) taken to establish correctness of prefix sum implementations, for increasing array lengths, on NVIDIA GPU, Intel CPU and ARM GPU architectures. The number of test cases associated with Voigtländer's method for each array length is also shown.

First Voigtländer shows, using relational parametricity, that a generic prefix sum is correct if and only if it behaves correctly for each length $n$ with respect to integer lists and list concatenation when applied to the input $[[0],[1], \ldots,[n-1]]$, that is, it yields the output $[[0],[0,1], \ldots,[0, \ldots, n-1]]$. Voigtländer states that this result was inspired by earlier unpublished work by Sheeran; Sheeran confirms this claim when exploiting the result in later work on the design of prefix sum algorithms [33]. We refer to this as the Sheeran result. The result also holds for fixed lengths $n$, which means the result is similar to our result for sequential prefix sums 
with respect to the interval monoid. However, the Sheeran result cannot be used for practical verification of prefix sums for large $n$, because $O\left(n^{2} \lg (n)\right)$ space is required to represent the result. ${ }^{10}$ Our interval of summations monoid avoids this problem by exploiting the fact that, as argued in Section 4, a correct generic prefix sum should never compute a non-contiguous summation. The interval of summations abstraction thus allows a contiguous summation to be represented precisely and compactly as an interval $(i, j)$ (or as $\mathbf{1}_{I}$ in the case of an empty summation), and collapses all other summations into the absorbing element $T$.

Letting $L$ denote the monoid of integer lists under list concatenation employed by the Sheeran method, there is an obvious homomorphism $\theta: L \rightarrow I$ defined by:

$$
\theta\left(\left[x_{1}, \ldots, x_{m}\right]\right)= \begin{cases}\mathbf{1}_{I} & \text { if } m=0 \\ \left(x_{1}, x_{m}\right) & \text { if } m>0 \text { and } \\ & \quad x_{i+1}=x_{i}+1(1 \leq i<m) \\ \top & \text { otherwise }\end{cases}
$$

This homomorphism discards exactly those summations that cannot contribute to a correct generic prefix sum.

Secondly, Voigtländer presents an elegant proof of the "0-1-2Principle" for parallel prefix computation. In the spirit of the 0-1Principle of Knuth [20], ${ }^{11}$ the 0-1-2-Principle states that a prefix sum algorithm is correct on all ternary sequences with every associative operator (defined over the ternary set) if and only if it is correct on any input sequence with an associative operator. In fact, carefully tracing his proof, Voigtländer observes that it suffices to consider just two operators and for every length $n$ a particular input set of ternary sequences of size $O\left(n^{2}\right)$ and $O(n)$ for his first and second operator, respectively. As with the Sheeran result, this result also holds for fixed lengths $n$. Voigtländer does not present an experimental evaluation of his method in [38]; we believe that our work is the first to do so. As we demonstrated in the experiments of Section 7, verifying a prefix sum by running Voigtländer's set of tests does not scale to large array sizes.

The focus of $[33,38]$ is on sequential HASKELL programs describing parallel synchronous hardware. In contrast, we have presented a method for verifying prefix sums implemented as asynchronous barrier-synchronising programs, leading to a practical method for verifying GPU kernel implementations. Due to our imperative, data-parallel setting, we present proofs using a direct simulation argument; we are not aware of any work on using relational parametricity to reason about data-parallel programs and believe this would be challenging.

Correct-by-derivation prefix sums An alternative approach by Hinze [17] is to construct prefix sums that are correct by derivation. In Hinze's work, rather than verifying candidate implementations for functional correctness, new prefix sum circuits are built from a set of basic primitives and combinators. The correctness of these circuits is ensured by a set of algebraic laws derived for these combinators. Similar to the work of Sheeran, prefix sums are given as HASKELL programs describing circuit layouts. We are not aware of any work that translates this approach to a data-parallel setting.

Formal verification of GPU kernels Recently a number of techniques for formal or semi-formal analysis of GPU kernels have been proposed [1, 9, 18, 23-25], and formal semantics for GPU kernels have been studied in-depth [10, 15]. The PUG [24] and GPUVerify [1] methods aim to statically prove race-freedom, while the

\footnotetext{
${ }^{10}$ The space complexity is $O\left(n^{2} \lg (n)\right)$ for correct algorithms; an incorrect algorithm could apply the concatenation operator arbitrarily many times, requiring unbounded space.

${ }^{11}$ If an oblivious sorting algorithm is correct on all Boolean valued input sequences then it is correct on input sequences over any totally ordered set.
}

method described in [23] uses a combination of dynamic and static analysis for race-freedom verification. We employed GPUVerify to prove race-freedom of prefix sum kernels in our experimental evaluation (Section 7). The GKLEE [25] and KLEE-CL [9] methods employ dynamic symbolic execution, based on the KLEE tool [6], to find data races and array bounds errors in CUDA and OpenCL kernels, respectively.

A recent technique for functional verification of OpenCL kernels using permission-based separation logic [18] could, in principle, be applied to generic prefix sum kernels. However, tool support for automation of this method is not yet available.

In prior work we introduced barrier invariants [8] to enable reasoning about data-dependent GPU kernels, and used barrier invariants to statically prove functional properties of Blelloch, BrentKung and Kogge-Stone prefix sum kernels. The challenges in making this approach scale provided insights which ultimately led to the interval of summations abstraction, and we used an early formulation of the abstraction to verify the Kogge-Stone kernel in [8]. The method for prefix sum verification presented here significantly outperforms the technique of [8], but the concept of barrier invariants has wider applicability.

\section{Conclusions}

We have introduced the interval of summations abstraction, investigated theoretical guarantees provided by this abstraction for verifying prefix sum algorithms, and shown that it can be used to design a practical and scalable verification method for GPU kernel implementations of prefix sums. Our experimental results demonstrate that we can prove race-freedom of prefix sum implementations for all conceivably useful array sizes, after which checking functional correctness of a prefix sum boils down to running a single test case, taking no longer than the time required to run the prefix sum in practice. We believe this substantiates our claim that we have made a major step towards solving the problem of functional verification for GPU implementations of generic prefix sums.

Our approach depends on the ability to prove that a data-parallel program behaves deterministically. For models of computation where barriers provide the only means of synchronisation this boils down to proving race-freedom (Lemma 5.4). Our approach thus extends to prefix sums written in programming models such as OpenMP, MPI and pthreads as long as these implementations use barriers exclusively for synchronisation, in favour of locks or atomic operations. Correspondingly, our method cannot be applied to GPU kernel implementations where threads communicate using atomics; in practice we have not seen the use of atomics in prefix sum kernels.

We have considered verification of prefix sums with respect to fixed array sizes. To prove that a prefix sum is correct for all array sizes it would be necessary to prove race-freedom for arbitrary array sizes, and also to prove that the prefix sum would compute the correct result for our interval of summations test case for every array size. While the former is beyond the scope of current tools such as GPUVerify, and the latter obviously cannot be achieved through testing, one could attempt to establish both through (manual or partially automated) mathematical proof.

We deliberately designed the interval of summations abstraction to capture the specific case of prefix sums, due to the importance of this class of algorithms. The abstraction can be used to analyse any algorithm that operates over an abstract monoid as long as the results computed by the algorithm are required to be contiguous summations. We are aware of one further class of such algorithms: reduction operations. The reduction of an array $\left[s_{1}, s_{2}, \ldots, s_{n}\right]$ is the sum $s_{1} \oplus s_{2} \oplus \cdots \oplus s_{n}$. Indeed, a reduction is performed during the first phase of the Blelloch and Brent-Kung prefix sum algorithms. 
A natural question is to ask if there are prefix sum algorithms that gain efficiency by exploiting other properties of operators, such as commutativity or idempotence. We are aware of recent work by Sergeev [32] which investigates prefix sum circuits for the XOR operator, exploiting the fact that this operator satisfies the identity $x \oplus y \oplus y=x$. Beyond prefix sums, there is scope for devising abstractions to allow representation-independent reasoning via a canonical test case in less restricted settings. For example, potentially discontiguous summations of monoid elements can be represented using lists $[33,38]$, and commutativity can be accommodated by switching to a bag representation. However, these representations lose the space-efficiency afforded by the interval of summations abstraction which allows our approach to scale.

\section{Acknowledgments}

We are grateful to Josh Berdine, Pantazis Deligiannis, Samin Ishtiaq, Alan Mycroft, Jakob Grue Simonsen, John Wickerson and our POPL reviewers for their insightful comments on various drafts of this work, and to Akash Lal for helpful discussion.

We thank Daniel Liew (Imperial College), Anton Lokhmotov (ARM) and Dong Ping Zhang (AMD) for their assistance with our experimental evaluation.

Finally, we thank Mary Sheeran and Janis Voigtländer for their work, which inspired our efforts.

\section{References}

[1] A. Betts, N. Chong, A. F. Donaldson, S. Qadeer, and P. Thomson. GPUVerify: a verifier for GPU kernels. In OOPSLA, pages 113-132, 2012.

[2] M. Billeter, O. Olsson, and U. Assarsson. Efficient stream compaction on wide SIMD many-core architectures. In $H P G$, pages 159-166, 2009.

[3] G. E. Blelloch. Scans as primitive parallel operations. IEEE Trans. Comput., 38(11):1526-1538, 1989.

[4] G. E. Blelloch. Prefix sums and their applications. In J. H. Reif, editor, Synthesis of Parallel Algorithms. Morgan Kaufmann, 1990.

[5] R. P. Brent and H.-T. Kung. A regular layout for parallel adders. IEEE Trans. Computers, 31(3):260-264, 1982.

[6] C. Cadar, D. Dunbar, and D. R. Engler. KLEE: Unassisted and automatic generation of high-coverage tests for complex systems programs. In OSDI, pages 209-224, 2008.

[7] S. Che et al. Rodinia: A benchmark suite for heterogeneous computing. In Workload Characterization, pages 44-54, 2009.

[8] N. Chong, A. F. Donaldson, P. H. Kelly, J. Ketema, and S. Qadeer. Barrier invariants: A shared state abstraction for the analysis of datadependent GPU kernels. In OOPSLA, pages 605-622, 2013.

[9] P. Collingbourne, , C. Cadar, and P. H. J. Kelly. Symbolic testing of OpenCL code. In HVC'11, pages 203-218, 2012.

[10] P. Collingbourne, A. F. Donaldson, J. Ketema, and S. Qadeer. Interleaving and lock-step semantics for analysis and verification of GPU kernels. In ESOP, pages 270-289, 2013.

[11] P. Cousot and R. Cousot. Abstract interpretation: A unified lattice model for static analysis of programs by construction or approximation of fixpoints. In POPL, pages 238-252, 1977.

[12] A. Danalis et al. The scalable heterogeneous computing (SHOC) benchmark suite. In GPGPU 2010, pages 63-74, 2010.

[13] O. Eğecioğlu, E. Gallopoulos, and C. Koç. A parallel method for fast and practical high-order Newton interpolation. BIT Numerical Mathematics, 30:268-288, 1990.
[14] W. Gropp, E. Lusk, and A. Skjellum. Using MPI: Portable Parallel Programming with the Message Passing Interface. MIT Press, 2nd edition, 1999.

[15] A. Habermaier and A. Knapp. On the correctness of the SIMT execution model of GPUs. In ESOP, pages 316-335, 2012.

[16] M. Harris, S. Sengupta, and J. Owens. Parallel prefix sum (scan) with CUDA. In H. Nguyen, editor, GPU Gems 3. Addison-Wesley, 2007.

[17] R. Hinze. An algebra of scans. In MPC, pages 186-210, 2004.

[18] M. Huisman and M. Mihelčić. Specification and verification of GPGPU programs using permission-based separation logic. In BYTECODE, 2013.

[19] Khronos OpenCL Working Group. The OpenCL specification, version 1.2, 2012.

[20] D. E. Knuth. The Art of Computer Programming, volume 3. AddisonWesley, 2nd edition, 1998.

[21] P. M. Kogge and H. S. Stone. A parallel algorithm for the efficient solution of a general class of recurrence equations. IEEE Trans. Computers, C-22(8):786-793, 1973.

[22] R. E. Ladner and M. J. Fischer. Parallel prefix computation. J. ACM, 27(4):831-838, 1980.

[23] A. Leung, M. Gupta, Y. Agarwal, et al. Verifying GPU kernels by test amplification. In PLDI, pages 383-394, 2012.

[24] G. Li and G. Gopalakrishnan. Scalable SMT-based verification of GPU kernel functions. In FSE, pages 187-196, 2010.

[25] G. Li, P. Li, G. Sawaya, G. Gopalakrishnan, I. Ghosh, and S. P. Rajan. GKLEE: concolic verification and test generation for GPUs. In $P P o P P$, pages 215-224, 2012.

[26] D. Merrill and A. Grimshaw. Parallel scan for stream architectures. Technical Report CX2009-14, Department of Computer Science, University of Virginia, 2009.

[27] NVIDIA. CUDA C programming guide, version 5.0, 2012.

[28] B. C. Pierce. Types and Programming Languages. MIT Press, 2002.

[29] P. Sanders and J. L. Träff. Parallel prefix (scan) algorithms for MPI. In PVM/MPI, pages 22-29, 2006.

[30] N. Satish, M. Harris, and M. Garland. Designing efficient sorting algorithms for manycore GPUs. In IPDPS, pages 1-10, 2009.

[31] S. Sengupta, M. Harris, Y. Zhang, and J. D. Owens. Scan primitives for GPU computing. In $G H$, pages 97-106, 2007.

[32] I. Sergeev. On the complexity of parallel prefix circuits. Technical Report TR13-041, Electronic Colloquium on Computational Complexity, 2013.

[33] M. Sheeran. Functional and dynamic programming in the design of parallel prefix networks. J. of Funct. Program., 21(1):59-114, 2011.

[34] J. Sklansky. Conditional-sum addition logic. IRE Trans. Electronic Computers, EC-9:226-231, 1960.

[35] B. So, A. M. Ghuloum, and Y. Wu. Optimizing data parallel operations on many-core platforms. In In First Workshop on Software Tools for Multi-Core Systems (STMCS), 2006.

[36] H. S. Stone. Parallel processing with the perfect shuffle. IEEE Trans. Comput., 20(2):153-161, 1971.

[37] J. Stratton et al. Parboil: A revised benchmark suite for scientific and commercial throughput computing. Technical Report IMPACT-12-01, University of Illinois at Urbana-Champaign, 2012.

[38] J. Voigtländer. Much ado about two (pearl): a pearl on parallel prefix computation. In POPL, pages 29-35, 2008. 